\title{
Improbability of Collisions in $n$-Body Systems
}

\author{
Stefan Fleischer and Andreas Knauf*
}

June 28, 2021

\begin{abstract}
For a wide class of two-body interactions, including standard examples like gravitational or Coulomb fields, we show that collision orbits in $n$-body systems are of Liouville measure zero for all energies. We use techniques from symplectic geometry to relate the volume of the union of collision orbits to the area of Poincaré surfaces surrounding the collision set.
\end{abstract}

\section{Introduction}

Consider as a primary example the motion of $n \in \mathbb{N}$ particles with masses $m_{1}, \ldots, m_{n}>0$ due to Newton's law of gravitation:

$$
m_{i} \ddot{q}_{i}=\sum_{j \in\{1, \ldots, n\} \backslash\{i\}} \frac{m_{j} m_{i}\left(q_{j}-q_{i}\right)}{\left\|q_{j}-q_{i}\right\|^{3}} \quad(i=1, \ldots, n) .
$$

Here we have set the scale of time in a way that the gravitational constant becomes 1 . For $n>1$ the flow of this ordinary differential equation is obviously not complete; for instance consider two particles, whose initial velocity vectors are pointing exactly towards each other - they will collide in finite time. Phase space points respectively their positive semi-orbits are called singular, if their maximal time interval of existence (for non-negative times) is finite. A singularity is called a collision, if all particles have limit positions in configuration space, as time approaches singular time, see Section 2 for further details.

In the papers [Saa71, Saa73], SAARI has shown the improbability of collisions, meaning that all collision points define a subset of phase space of Lebesgue measure zero. His techniques can be used to generalize this result to a class

*Department of Mathematics, Friedrich-Alexander-University Erlangen-Nürnberg, Cauerstr. 11, D-91058 Erlangen, Germany, fleischer, knauf@math.fau.de 
of homogeneous force fields including the gravitational case. As he points out, however, his bounds are not optimal.

Here, we generalize the result to an even wider class of potentials, also implementing optimal bounds. By giving up assumptions like homogeneity of the force field, we cannot rely on certain arguments any longer, for example arising from the Lagrange-Jacobi-Identity. Instead, we employ geometric techniques:

The first is based on a decomposition of configuration space, invented by GianMichele Graf in showing asymptotic completeness of quantum scattering.

The second comes from symplectic geometry: after defining an appropriate sequence of hypersurfaces surrounding the collision set, we can relate their surface area to the volume of the set of initial points, whose orbits are passing through the surfaces. The technical aspects of this method have been worked out in [FK18].

The outline of the paper is as follows: in Section 2 we define our class of admissible potentials and state the main result, Theorem 2.5. Section 3 presents an adapted partition of configuration space and a sequence of hypersurfaces onto which the Poincare surfaces project. In Section 4, by building on the main technique introduced in [FK18], we give the definition of a sequence of Poincare surfaces. Then we estimate their symplectic (as opposed to Riemannian) volume in Proposition 4.7. In Proposition 5.6 of Section 5 we prove finiteness of the time integral of kinetic energy. In Section 6 the Poincaré surfaces are then shown to eventually be hit by every collision orbit (Proposition 6.1).

In combination, Propositions 4.7 and 6.1, together with Theorem A of [FK18], imply our main result, Theorem 2.5 .

In the final section 7 we indicate the minimal changes that need to be done in order to prove analogous theorems

- for multiple collisions on the line $(d=1)$, or

- in the presence of fixed centers.

General Notation: We point out that we use $C$ as a positive constant, that only depends on fixed system parameters like number of particles or their masses. Its value may change with every usage, even within the same set of equations.

\section{Statement of the Main Result}

We consider the motion of $n \geq 2$ particles with respective masses $m_{1}, \ldots, m_{n}$ in $d \geq 2$ dimensions. Thus the (a priori) configuration space is given by

$$
M:=M_{1} \oplus \ldots \oplus M_{n}
$$


with $M_{k}:=\mathbb{R}^{d}$ for $k \in N:=\{1, \ldots, n\}$. We write the elements of $M$ in the form

$$
q=\left(q_{1}, \ldots, q_{n}\right) .
$$

Using the canonical identification $T^{*} M=M \times M^{*}$ with $M^{*}$ being the vector space dual to $M$, we write the elements of the cotangent bundle $T^{*} M$ in the form

$$
x=(q, p)=\left(q_{1}, \ldots, q_{n}, p_{1}, \ldots, p_{n}\right) .
$$

The inner product on $M$ defined by

$$
\langle\cdot, \cdot\rangle_{M}: M \times M \rightarrow \mathbb{R} \quad, \quad\left\langle q, q^{\prime}\right\rangle_{M}:=\left\langle q, \mathcal{M} q^{\prime}\right\rangle,
$$

where $\langle\cdot, \cdot\rangle$ is the Euclidean inner product on $M$ and

$$
\mathcal{M}:=\operatorname{diag}\left(m_{1}, \ldots, m_{n}\right) \otimes \mathbb{1}_{d}
$$

is the scaling according to the masses, induces an inner product on $M^{*}$ via

$$
\langle\cdot, \cdot\rangle_{M^{*}}: M^{*} \times M^{*} \rightarrow \mathbb{R} \quad, \quad\left\langle p, p^{\prime}\right\rangle_{M^{*}}:=\left\langle p, \mathcal{M}^{-1} p^{\prime}\right\rangle
$$

as well as an inner product on the cotangent bundle $T^{*} M$ via

$$
\langle\cdot, \cdot\rangle_{T^{*} M}: T^{*} M \times T^{*} M \rightarrow \mathbb{R}, \quad\left\langle x, x^{\prime}\right\rangle_{T^{*} M}:=\left\langle q, q^{\prime}\right\rangle_{M}+\left\langle p, p^{\prime}\right\rangle_{M^{*}} .
$$

By this, we get a Riemannian manifold $\left(T^{*} M, g\right)$ with $g \equiv\langle\cdot, \cdot\rangle_{T^{*} M}$, the Riemannian volume form of which is given by the symplectic volume form $\Omega_{n d}$, with

$$
\Omega_{k}:=\frac{(-1)^{\lfloor k / 2\rfloor}}{k !} \omega^{\wedge k} \quad(k=1, \ldots, n d) .
$$

Here $\omega$ is the canonical symplectic form on $T^{*} M . \mathcal{M}$ induces an identification $T^{*} M \cong \mathbb{R}^{2 n d}$, the Riemannian volume form is equal to Lebesgue measure $\lambda^{2 n d}$. We will use that $\left(T^{*} M, g, \omega\right)$ is a Kähler manifold.

The force field defining the motion consists of two-body interactions. We write

$$
\Delta:=\left\{q \in M \mid \text { there exist } i \neq j \in N \text { with } q_{i}=q_{j}\right\}
$$

for the collision set. Thus the (actual) configuration space $\widehat{M}$ is defined by

$$
\widehat{M}:=M \backslash \Delta \text {. }
$$

On phase space $\widehat{P}:=T^{*} \widehat{M}$, the Hamiltonian function is defined by

$$
H \in C^{2}(\widehat{P}, \mathbb{R}), \quad H(q, p):=K(p)+V(q) .
$$


Here,

$$
K: T^{*} M \rightarrow \mathbb{R}, \quad K(q, p) \equiv K(p)=\frac{1}{2}\langle p, p\rangle_{M^{*}}=\sum_{k \in N} \frac{\left\|p_{k}\right\|^{2}}{2 m_{k}}
$$

is the kinetic energy and $V: \widehat{M} \rightarrow \mathbb{R}$ is the potential; we assume the potential to be of the form

$$
V(q)=\sum_{i<j \in N} V_{i, j}\left(q_{i}-q_{j}\right)
$$

with two-body potentials $V_{i, j} \in C^{2}\left(\mathbb{R}^{d} \backslash\{0\}, \mathbb{R}\right)$. For simplifying notation, we write

$$
V_{j, i}(q)=V_{i, j}(-q) \quad\left(1 \leq i<j \leq n, q \in \mathbb{R}^{d} \backslash\{0\}\right) .
$$

The Hamiltonian vector field $X_{H}$ is defined by the equation $\boldsymbol{i}_{X_{H}} \omega=\boldsymbol{d} H$, where $\boldsymbol{i}$ is the inner product and $\boldsymbol{d}$ is the exterior derivative. So it is continuously differentiable, and the Hamiltonian differential equation $\dot{x}=X_{H}(x)$ has local solutions. In coordinates, the differential equation is given by

$$
\dot{q}_{k}=\frac{p_{k}}{m_{k}} \quad, \quad \dot{p}_{k}=-\sum_{i \in N \backslash\{k\}} \nabla V_{k, i}\left(q_{k}-q_{i}\right) \quad(k \in N) .
$$

Definition 2.1 We call the potential $V$ admissible, if $\lim _{\|q\| \rightarrow \infty} V_{i, j}(q)=0$, there exists an $\alpha \in(0,2)$ such that

$$
D^{2} V_{i, j}(q)=\mathcal{O}\left(\|q\|^{-\alpha-2}\right) \quad(\|q\| \leq 1)
$$

and for some $C_{V}>0$ either

1. for suitable $Z_{i, j} \in \mathbb{R}$

$$
\left|\left\langle\frac{q}{\|q\|}, \nabla V_{i, j}(q)\right\rangle+\alpha \frac{Z_{i, j}}{\|q\|^{\alpha+1}}\right| \leq C_{V} \quad(\|q\| \in(0,1], 1 \leq i<j \leq n)
$$

2. or the $V_{i, j}$ are bounded above, and, with $W_{-}(q):=\max (-W(q), 0)$,

$$
\left\langle q, \nabla V_{i, j}(q)\right\rangle \leq C_{V}+\alpha\left(V_{i, j}\right)_{-}(q) \quad(\|q\| \in(0,1], 1 \leq i<j \leq n) .
$$

\section{Example 2.2 (Admissible Potentials)}

1. An important class of admissible potentials consists of the homogeneous potentials

$$
V_{i, j}(q)=\frac{Z_{i, j}}{\|q\|^{\alpha_{i, j}}}
$$


with $Z_{i, j} \in \mathbb{R}$ and $\alpha_{i, j}=\alpha \in(0,2)$, or with $Z_{i, j}<0$ and $\alpha_{i, j} \in(0,2)$.

In particular, this includes the cases of gravitational and Coulomb force fields. ${ }^{1}$

Note that collisions of three or more charged particles are possible, although some of them then necessarily repel each other.

Perhaps the easiest case is the one of a particle with charge $\rho_{1}>0$ resting at the origin and two particles with masses $m_{2}=m_{3}$ and charges $\rho_{2}=\rho_{3} \in$ $\left(-4 \rho_{1}, 0\right)$, with positions $q_{2}=-q_{3}$ and momenta $p_{2}=-p_{3}$.

2. (2.10) also includes the physically important case of Yukawa potentials

$$
V_{i, j}(q)=Z_{i, j} \frac{\exp \left(-m_{i, j}\|q\|\right)}{\|q\|} \quad\left(Z_{i, j} \in \mathbb{R}, m_{i, j}>0\right) .
$$

Then

$$
\left|\left\langle\frac{q}{\|q\|}, \nabla V_{i, j}(q)\right\rangle+\alpha \frac{Z_{i, j}}{\|q\|^{\alpha+1}}\right|=\frac{Z_{i, j}}{\|q\|^{2}}\left(1-e^{-m_{i, j}\|q\|}\right)\left(m_{i, j}\|q\|+1\right)=\mathcal{O}(1) . \diamond
$$

\section{Remark 2.3 (Admissible Potentials)}

Aside for allowing for positive and negative singularities, Condition (2.10) is much stricter than (2.11), as up to a constant the potentials $V_{i, j}$ are homogeneous near zero: (2.10) entails for $q \in \mathbb{R}^{d} \backslash\{0\}$ with $\|q\| \leq 1$

$$
\begin{aligned}
V_{i, j}(q) & =V_{i, j}(q /\|q\|)-\int_{1}^{\|q\|^{-1}} \frac{d}{d s} V_{i, j}(s q) d s \\
& \leq V_{i, j}(q /\|q\|)+\int_{1}^{\|q\|^{-1}}\left(\frac{Z_{i, j}}{\|q\|^{\alpha}} \alpha s^{-(\alpha+1)}+C_{V}\|q\|\right) d s \\
& =V_{i, j}(q /\|q\|)+C_{V}(1-\|q\|)-Z_{i, j}+\frac{Z_{i, j}}{\|q\|^{\alpha}},
\end{aligned}
$$

and similarly for the other direction of the inequality. So by compactness of $S^{d-1}$

$$
\left|V_{i, j}(q)-\frac{Z_{i, j}}{\|q\|^{\alpha}}\right| \leq\left|V_{i, j}(q /\|q\|)\right|+\left|Z_{i, j}\right|+C_{V}(1-\|q\|)=\mathcal{O}(1) .
$$

For homogeneous attracting potentials proofs simplify, since one can make use of the results of POLLARD and SAARI in [PS68].

\footnotetext{
${ }^{1}$ With $\alpha_{i, j}=1$ and $Z_{i, j}:=-m_{i} m_{j}$ for all $1 \leq i<j \leq n$, we get the case of interaction due to gravitation, cf. (1.1). With $Z_{i, j}:=\rho_{i} \rho_{j}$ (here, the particles' charges $\rho_{k} \in \mathbb{R}$ take over the role of the particles' masses) we get the case of interaction due to static electrical charge.
} 
Going back to the Hamiltonian system as defined above, the corresponding Hamiltonian flow $\Phi: D \rightarrow \widehat{P}$ uniquely exists on a maximal neighborhood $D \subseteq \mathbb{R} \times \widehat{P}$ of $\{0\} \times \widehat{P}$ in extended phase space; we have $\Phi \in C^{1}(D, \widehat{P})$. Shortly, we write

$$
\Phi(t, x)=\Phi_{t}(x)=(q(t, x), p(t, x))=(q(t), p(t)) \quad((t, x) \in D),
$$

the latter if there is no ambiguity concerning the initial condition $x \in \widehat{P}$. The flow's domain of definition is of the form

$$
D=\left\{(t, x) \in \mathbb{R} \times \widehat{P} \mid T^{-}(x)<t<T^{+}(x)\right\}
$$

with the escape time $T=T^{+}: \widehat{P} \rightarrow(0, \infty]$; by reversibility of $X_{H}$ we have $T^{-}(q, p)=-T^{+}(q,-p)$. Additionally, $T^{+}$is lower semi-continuous. By

$$
\text { Sing }:=\{x \in \widehat{P} \mid T(x)<\infty\},
$$

we denote the set of phase space points experiencing a singularity.

In celestial mechanics, it is a well known fact due to Painlevé, that a singularity occurs if and only if the minimal particle distance converges to zero. As a first result, we point out that this still holds in our more general setting of two-body interactions, since the classical proof can be applied. For this purpose, let

$$
q_{\min }: \widehat{P} \rightarrow(0, \infty) \quad, \quad(q, p) \mapsto \min \left\{\left\|q_{i}-q_{j}\right\| \mid i \neq j \in N\right\} .
$$

be the minimal distance of particles. Then we get:

Theorem 2.4 (Painlevé) Let $x \in$ Sing. Then $\lim _{t \nearrow T(x)} q_{\min } \circ \Phi_{t}(x)=0$.

Proof: Otherwise, there exist $\delta>0$ and a sequence of monotonically increasing times $\left(s_{j}\right)_{j \in \mathbb{N}}$ with $\lim _{j \rightarrow \infty} s_{j}=T(x)$ and $\operatorname{dist}\left(q\left(s_{j}\right), \Delta\right)>\delta$. By assumption, the potential is bounded below on the domain

$$
U:=\left\{q \in \widehat{M} \mid \operatorname{dist}(q, \Delta) \geq \frac{\delta}{2}\right\},
$$

that is $V_{\min }:=\inf _{q \in U} V(q) \in \mathbb{R}$. Thus by conservation of energy $E:=H(x)$, as long as $q(t) \in U$, velocity is bounded above, namely

$$
\|\dot{q}(t)\| \leq v_{\max }:=\sqrt{2 m_{\max }\left(E-V_{\min }\right)}
$$

with $m_{\max }:=\max \left\{m_{1}, \ldots, m_{n}\right\}$. So for all $j \in \mathbb{N}$ the solution can be extended at least up to $s_{j}+\frac{\delta}{2 v_{\max }}$. This contradicts the assumption $\lim _{j \rightarrow \infty} s_{j}=T(x)$.

Within this work, we are particularly interested in those singularities, which have 
limit positions in configuration space at singular time, and call them collision singularities:

$$
\text { Coll } \left.:=\left\{x \in \operatorname{Sing} \mid \lim _{t \nearrow T(x)} q(t, x) \text { exists (in } M\right)\right\} .
$$

Furthermore, we restrict considerations to the energy surfaces

$$
\Sigma_{E}:=H^{-1}(E) \quad(E \in \mathbb{R}) .
$$

Since Sing is a subset of the open domain consisting of all non-equilibrium points in $\widehat{P}$, we can without loss of generality assume that every $E \in \mathbb{R}$ is a regular value of $H$. Thus, $\imath_{E}: \Sigma_{E} \rightarrow \widehat{P}$ is a codimension one submanifold (if non-empty). We write $\mathrm{Coll}_{E}:=\operatorname{Coll} \cap \Sigma_{E}$.

There is a $(2 n d-1)$-form $\sigma$ on phase space $\widehat{P}$ with $d H \wedge \sigma=\Omega_{n d}$, see Remark 1.4 of [FK18]. Although $\sigma$ is not fixed by that property, its pull-back $\sigma_{E}:=\imath_{E}^{*} \sigma$ is a uniquely defined volume form on that energy surface, invariant under the restricted flow. We denote by $\sigma_{E}$, too the corresponding Liouville measure on $\Sigma_{E}$. Now we can state our main result:

Theorem 2.5 For all $n \in \mathbb{N}, d \geq 2$ and $E \in \mathbb{R}$ the set Coll $_{E}$ of phase space points leading to a collision has Liouville measure zero, provided $V$ is admissible.

By integration with respect to total energy $E$ it follows that the Lebesgue measure $\lambda^{2 d n}$ (Coll) of the collision set in phase space $\widehat{P}$ vanishes, too.

\section{Partitioning Configuration Space}

\section{Cluster Coordinates}

We now introduce coordinates, that (notationally) link certain subgroups of particles together, in the form of so-called clusters. The external cluster coordinates then describe the motion of the cluster as a whole, whereas the internal ones describe each particle's motion within its cluster.

We begin with some standard notions:

\section{Definition 3.1}

- A set partition or cluster decomposition of $N$ is a set $\mathcal{C}:=\left\{C_{1}, \ldots, C_{k}\right\}$ of blocks or clusters $\emptyset \neq C_{\ell} \subseteq N$ such that

$$
\bigcup_{\ell=1}^{k} C_{\ell}=N \quad \text { and } \quad C_{\ell} \cap C_{m}=\emptyset \text { for } \ell \neq m \text {. }
$$

We denote by $\sim_{\mathcal{C}}$ (or $\sim$, if there is no ambiguity) the equivalence relation on $N$ induced by $\mathcal{C}$; the corresponding equivalence classes are denoted by $[\cdot]_{\mathcal{C}}$. 
- The lattice of partitions $\mathcal{P}(N)$ is the set of cluster decompositions $\mathcal{C}$ of $N$, partially ordered by refinement, i.e.,

$$
\mathcal{C}=\left\{C_{1}, \ldots, C_{k}\right\} \preccurlyeq\left\{D_{1}, \ldots, D_{\ell}\right\}=\mathcal{D},
$$

if $C_{m} \subseteq D_{\pi(m)}$ for an appropriate mapping $\pi:\{1, \ldots, k\} \rightarrow\{1, \ldots, \ell\}$. In this case, $\mathcal{C}$ is called finer than $\mathcal{D}$ and $\mathcal{D}$ coarser than $\mathcal{C}$.

The unique finest and coarsest elements of $\mathcal{P}(N)$ are

$$
\mathcal{C}_{\min }:=\{\{1\}, \ldots,\{n\}\} \quad \text { and } \mathcal{C}_{\max }:=\{N\}=\{\{1, \ldots, n\}\},
$$

respectively. By $\mathcal{P}_{0}(N)$ we denote the set of all decompositions without the finest one, i.e. $\mathcal{P}_{0}(N):=\mathcal{P}(N) \backslash\left\{\mathcal{C}_{\min }\right\}$.

- The rank of $\mathcal{C} \in \mathcal{P}(N)$ is the number $|\mathcal{C}|$ of its blocks.

- The join of $\mathcal{C}$ and $\mathcal{D} \in \mathcal{P}(N)$, denoted as $\mathcal{C} \vee \mathcal{D}$, is the finest cluster decomposition that is coarser than both $\mathcal{C}$ and $\mathcal{D}$.

We use partitions to decompose configuration space: given a subset $\emptyset \neq C \subseteq N$, we define the corresponding collision set as

$$
\Delta_{C}^{E}:=\left\{q \in M \mid q_{i}=q_{j} \text { if } i, j \in C\right\},
$$

and for a cluster decomposition $\mathcal{C}$ we define the $\mathcal{C}$-collision subspace

$$
\Delta_{\mathcal{C}}^{E}:=\left\{q \in M \mid q_{i}=q_{j} \text { if }[i]_{\mathcal{C}}=[j]_{\mathcal{C}}\right\}=\bigcap_{C \in \mathcal{C}} \Delta_{C}^{E}
$$

By $\Pi_{C}^{E}$ we denote the $\mathcal{M}$-orthogonal projection onto the subspace $\Delta_{C}^{E}$, and we denote the complementary projection $\mathbb{1}_{\mathcal{M}}-\Pi_{C}^{E}$ by $\Pi_{C}^{I}$. Accordingly, we denote the projection onto $\Delta_{\mathcal{C}}^{E}$ by $\Pi_{\mathcal{C}}^{E}:=\prod_{C \in \mathcal{C}} \Pi_{C}^{E}$, and the complementary projection by $\Pi_{\mathcal{C}}^{I}=\mathbb{1}_{\mathcal{M}}-\Pi_{\mathcal{C}}^{E}=\sum_{C \in \mathcal{C}} \Pi_{C}^{I}$. The image of $\Pi_{C}^{I}$ then is given by

$$
\Delta_{C}^{I}:=\left\{q \in M \mid \sum_{i \in C} m_{i} q_{i}=0, \forall i \in N \backslash C: q_{i}=0\right\},
$$

the image of $\Pi_{\mathcal{C}}^{I}$ is given by

$$
\Delta_{\mathcal{C}}^{I}:=\left\{q \in M \mid \forall C \in \mathcal{C}: \sum_{i \in C} m_{i} q_{i}=0\right\}=\bigoplus_{C \in \mathcal{C}} \Delta_{C}^{I} .
$$

In particular, $\Delta_{\mathcal{C}_{\min }}^{E}=M$. Regarding the dimensions of these subspaces, we have

$$
\begin{aligned}
\operatorname{dim}\left(\Delta_{\mathcal{C}}^{E}\right) & =d\left(n-\sum_{C \in \mathcal{C}}(|C|-1)\right)=d|\mathcal{C}|, \\
\operatorname{dim}\left(\Delta_{\mathcal{C}}^{I}\right) & =d \sum_{C \in \mathcal{C}}(|C|-1)=d(n-|\mathcal{C}|) .
\end{aligned}
$$


Thus we get a $\mathcal{M}$-orthogonal decomposition

$$
M=\Delta_{\mathcal{C}}^{E} \oplus \bigoplus_{C \in \mathcal{C}} \Delta_{C}^{I}
$$

For a nonempty subset $C \subseteq N$ we define the cluster mass, cluster barycenter and cluster momentum of $C$ by

$$
m_{C}:=\sum_{j \in C} m_{j} \quad, \quad q_{C}:=\frac{1}{m_{C}} \sum_{j \in C} m_{j} q_{j} \quad \text { and } \quad p_{C}:=\sum_{i \in C} p_{i} .
$$

In particular $m_{N}$ equals the total mass of the particle system. Then for the partitions $\mathcal{C} \in \mathcal{P}(N)$ the $i$-th component of the cluster projection $q_{\mathcal{C}}^{E}:=\Pi_{\mathcal{C}}^{E}(q)$ is given by the barycenter

$$
\left(q_{\mathcal{C}}^{E}\right)_{i}=q_{[i]_{\mathcal{C}}} \quad(i \in N)
$$

of its cluster. Similarly for $q_{\mathcal{C}}^{I}:=\Pi_{\mathcal{C}}^{I}(q)$,

$$
\left(q_{\mathcal{C}}^{I}\right)_{i}=q_{i}-q_{[i]_{\mathcal{C}}} \quad(i \in N)
$$

is its distance from the barycenter.

Join of partitions corresponds to intersection of collision subspaces:

$$
\Delta_{\mathcal{C}}^{E} \cap \Delta_{\mathcal{D}}^{E}=\Delta_{\mathcal{C} \vee \mathcal{D}}^{E} \quad(\mathcal{C}, \mathcal{D} \in \mathcal{P}(N))
$$

So the mutually disjoint sets

$$
\Xi_{\mathcal{C}}^{(0)}:=\Delta_{\mathcal{C}}^{E} \backslash \bigcup_{\mathcal{D} \nsucceq \mathcal{C}} \Delta_{\mathcal{D}}^{E} \quad(\mathcal{C} \in \mathcal{P}(N)),
$$

form a set partition of $M$, with $\Xi_{\mathcal{C}_{\min }}^{(0)}=\widehat{M}$. Note that we can write $\Xi_{\mathcal{C}}^{(0)}$ as $\Xi_{\mathcal{C}}^{(0)}=\left\{q \in M \mid q_{i}=q_{j}\right.$ if and only if $\left.i \sim_{\mathcal{C}} j\right\}$. Based on this, we partition the collision set Coll $\subseteq \widehat{P}$ uniquely into clusters by

$$
\mathrm{SP}: \text { Coll } \rightarrow \mathcal{P}_{0}(N) \quad, \quad \lim _{t \nearrow T(x)} q(t, x) \in \Xi_{\mathrm{SP}(x)}^{(0)} .
$$

\section{The Graf Partition}

This partition, introduced by G.-M. Graf, relies on the (mean) moment of inertia

$$
J: M \rightarrow \mathbb{R} \quad, \quad J(q)=\|q\|_{\mathcal{M}}^{2}=\sum_{k=1}^{n} m_{k}\left\|q_{k}\right\|^{2},
$$

see Dereziński and GÉrard [DG97, Chapter 5], and [Kn18, Chapter 12.6]. 
Lemma 3.2 In the cluster decomposition $\mathcal{C} \in \mathcal{P}(N), J$ is of the form

$$
J=J_{\mathcal{C}}^{E}+J_{\mathcal{C}}^{I} \quad \text { with } \quad J_{\mathcal{C}}^{E}:=J \circ \Pi_{\mathcal{C}}^{E} \quad \text { and } \quad J_{\mathcal{C}}^{I}:=J \circ \Pi_{\mathcal{C}}^{I} .
$$

For $\mathcal{C} \preccurlyeq \mathcal{D}, J_{\mathcal{C}}^{E}-J_{\mathcal{D}}^{E} \geq 0$ is a quadratic form with index of inertia $d(|\mathcal{C}|-|\mathcal{D}|)$.

Proof: Indeed, $J(q)=\left\langle\left(\Pi_{\mathcal{C}}^{E}+\Pi_{\mathcal{C}}^{I}\right) q,\left(\Pi_{\mathcal{C}}^{E}+\Pi_{\mathcal{C}}^{I}\right) q\right\rangle_{\mathcal{M}}$, and

$$
\left\langle\Pi_{\mathcal{C}}^{E} q, \Pi_{\mathcal{C}}^{I} q\right\rangle_{\mathcal{M}}=\left\langle\Pi_{\mathcal{C}}^{E} q,\left(\mathbb{1}_{\mathcal{M}}-\Pi_{\mathcal{C}}^{E}\right) q\right\rangle_{\mathcal{M}}=\left\langle q, \Pi_{\mathcal{C}}^{E}\left(\mathbb{1}_{\mathcal{M}}-\Pi_{\mathcal{C}}^{E}\right) q\right\rangle_{\mathcal{M}}=0 .
$$

$J_{\mathcal{C}}^{E}(q)-J_{\mathcal{D}}^{E}(q)=\left\langle\left(\Pi_{\mathcal{C}}^{E}-\Pi_{\mathcal{D}}^{E}\right) q, q\right\rangle_{\mathcal{M}}$ with $\Pi_{\mathcal{C}}^{E} \Pi_{\mathcal{D}}^{E}=\Pi_{\mathcal{D}}^{E}$, and so the formula for the index of positive inertia follows from (3.2).

\section{Remark 3.3 (Moments of Inertia) Here}

1. $J_{\mathcal{C}}^{E}(q)$ equals the moment of inertia of the configuration in which all masses of each cluster are joined in its center of mass. By Lemma 3.2 the index of inertia of this quadratic form equals $d|\mathcal{C}|$;

2. $J_{\mathcal{C}}^{I}(q)$ is the sum of the moments of inertia of the clusters, each referred to the respective center of mass, rather than the origin;

3. For $\mathcal{C} \preccurlyeq \mathcal{D}$ with $|\mathcal{C}|=|\mathcal{D}|+1$, there is a unique cluster $D \in \mathcal{D}$ which is the disjoint union $C_{1} \dot{\cup} C_{2}$ of two clusters $C_{1}, C_{2} \in \mathcal{C}$, and the other clusters of $\mathcal{D}$ coincide with the other clusters of $\mathcal{C}$. Then

$$
\begin{aligned}
J_{\mathcal{C}}^{E}(q)-J_{\mathcal{D}}^{E}(q) & =J_{C_{1}}^{E}(q)+J_{C_{2}}^{E}(q)-J_{D}^{E}(q) \\
& =m_{C_{1}}\left\langle q_{C_{1}}, q_{C_{1}}\right\rangle+m_{C_{2}}\left\langle q_{C_{2}}, q_{C_{2}}\right\rangle-m_{D}\left\langle q_{D}, q_{D}\right\rangle \\
& =\frac{m_{C_{1}} m_{C_{2}}}{m_{D}}\left\|q_{C_{1}}-q_{C_{2}}\right\|^{2} .
\end{aligned}
$$

This measures the squared distance of the barycenters of $C_{1}$ and $C_{2}$.

Similar statements are true for the external kinetic energies, that is, the quadratic forms $K_{\mathcal{C}}^{E}: M^{*} \rightarrow \mathbb{R} \quad(\mathcal{C} \in \mathcal{P}(N))$.

Definition 3.4 For $\delta \in(0,1)$ and $k>0$, let

$$
J^{(k)}: M \rightarrow \mathbb{R} \quad, \quad J^{(k)}(q):=\max \left\{J_{\mathcal{C}}^{E}(q)+k \delta^{|\mathcal{C}|} \mid \mathcal{C} \in \mathcal{P}(N)\right\} .
$$

The Graf partition of the configuration space $M$ is the family of subsets

$$
\Xi_{\mathcal{C}}^{(k)}:=\left\{q \in M \mid J_{\mathcal{C}}^{E}(q)+k \delta^{|\mathcal{C}|}=J^{(k)}(q)\right\} \quad(\mathcal{C} \in \mathcal{P}(N)) .
$$




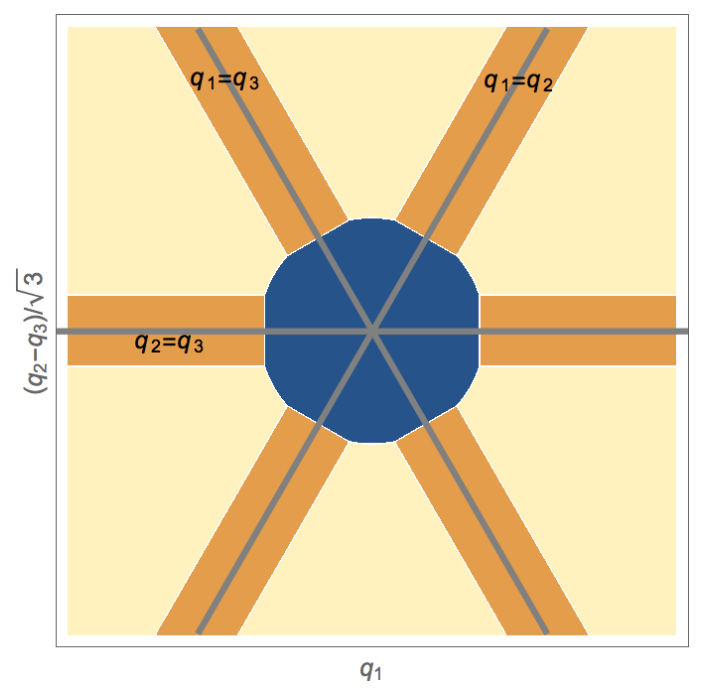

Figure 1: Graf partition of the configuration space (center of mass at 0 ) for $n=3$ particles in $d=1$ dimension. Yellow: $\Xi_{\mathcal{C}_{\min }}^{(k)}$, Blue: $\Xi_{\mathcal{C}_{\max }}^{(k)}$; from [Kn18].

The dependence on the parameter $k$ is homogeneous: In Minkowski notation

$$
\Xi_{\mathcal{C}}^{(k)}=k^{1 / 2} \Xi_{\mathcal{C}}^{(1)} \quad(\mathcal{C} \in \mathcal{P}(N), k>0) .
$$

The Graf partition is a measure theoretic partition of $M$ with respect to Lebesgue measure, i.e. for $\mathcal{C} \neq \mathcal{D}$ one has $\lambda^{n d}\left(\Xi_{\mathcal{C}}^{(k)} \cap \Xi_{\mathcal{D}}^{(k)}\right)=0$.

For small $\delta \in(0,1)$ (and, by (3.10), all $k>0$ ), the Graf partition (3.9) has the property that for $\Xi_{\mathcal{C}}^{(k)} \cap \Xi_{\mathcal{D}}^{(k)} \neq \emptyset$, the cluster decompositions $\mathcal{C}$ and $\mathcal{D}$ are comparable, i.e., $\mathcal{C} \preccurlyeq \mathcal{D}$ or $\mathcal{C} \succcurlyeq \mathcal{D}$. See [Kn18, Lemma 12.52] for a proof.

In Figure 1 we show a Graf partition.

We need quantitative estimates for intracluster and intercluster distances:

Lemma 3.5 For small enough $\delta \in\left(0, \frac{1}{2}\right)$ in Definition 3.4 there exist constants $C^{I}, C^{E}>0$ with $C^{I} \leq C^{E} / 4$ such that for all $\mathcal{C} \in \mathcal{P}_{0}(N)$

$$
\begin{array}{cl}
\left\|q_{i}^{I}\right\| \leq C^{I} \sqrt{k} & \left(i \in N, q \in \Xi_{\mathcal{C}}^{(k)}\right), \\
\left\|q_{C_{i}}-q_{C_{j}}\right\| \geq C^{E} \sqrt{k} \quad\left(C_{i} \neq C_{j} \in \mathcal{C}, q \in \Xi_{\mathcal{C}}^{(k)}\right),
\end{array}
$$

and thus

$$
\left\|q_{i}-q_{j}\right\| \geq \frac{1}{2}\left\|q_{[i]_{\mathcal{C}}}-q_{[j]_{\mathcal{C}}}\right\| \quad\left([i]_{\mathcal{C}} \neq[j]_{\mathcal{C}}, q \in \Xi_{\mathcal{C}}^{(k)}\right) .
$$

\section{Proof:}


- To prove (3.11), we note that by definition (3.9) of $\Xi_{\mathcal{C}}^{(k)}$ we have

$$
J_{\mathcal{C}}^{I}(q)=J(q)-J_{\mathcal{C}}^{E}(q)=J_{\mathcal{C}_{\min }^{E}}^{E}(q)-J_{\mathcal{C}}^{E}(q) \leq k\left(\delta^{|\mathcal{C}|}-\delta^{n}\right) \quad\left(q \in \Xi_{\mathcal{C}}^{(k)}\right) .
$$

Now $m_{i}\left\|q_{i}^{I}\right\|^{2} \leq J_{\mathcal{C}}^{I}(q)$ and $|\mathcal{C}| \leq n-1$, so that with $C^{I}:=\left(\frac{\delta^{n-1}}{2 m_{\min }}\right)^{1 / 2}$ inequality (3.11) follows.

- For (3.12) we compare $\mathcal{C}=\left\{C_{1}, \ldots, C_{\ell}\right\}$ with

$$
\mathcal{D}:=\left\{C_{r} \mid r \in\{1, \ldots, \ell\} \backslash\{i, j\}\right\} \dot{\cup}\{D\} \quad \text {, with } \quad D:=C_{i} \cup C_{j} .
$$

So $\mathcal{D} \succcurlyeq \mathcal{C}$ with $|\mathcal{D}|=|\mathcal{C}|-1 \leq n-2$. By Remark 3.3.3

$$
\frac{m_{C_{i}} m_{C_{j}}}{m_{D}}\left\|q_{C_{i}}-q_{C_{j}}\right\|^{2}=J_{\mathcal{C}}^{E}(q)-J_{\mathcal{D}}^{E}(q) \geq k\left(\delta^{|\mathcal{D}|}-\delta^{|\mathcal{C}|}\right) \geq k \delta^{n-2} / 2 .
$$

As $m_{D}=m_{C_{i}}+m_{C_{j}},\left\|q_{C_{i}}-q_{C_{j}}\right\| \geq C^{E} \sqrt{k}$ with $C^{E}:=\left(\frac{\delta^{n-2}}{2 n m_{\max }}\right)^{1 / 2}$.

- So for $\delta>0$ small, $C^{I} \leq C^{E} / 4$. Now (3.13) follows by the triangle inequality $\left\|q_{i}-q_{j}\right\| \geq\left\|q_{[i]_{\mathcal{C}}}-q_{[j]_{\mathcal{C}}}\right\|-\left\|q_{i}^{I}\right\|-\left\|q_{j}^{I}\right\| \geq\left\|q_{[i]_{\mathcal{C}}}-q_{[j]_{\mathcal{C}}}\right\|-\frac{C^{E}}{2} \sqrt{k} \geq \frac{1}{2}\left\|q_{[i]_{\mathcal{C}}}-q_{[j]_{\mathcal{C}}}\right\| . \square$

The sets

$$
\Xi^{(k)}:=\bigcup_{\mathcal{C} \in \mathcal{P}_{0}(N)} \Xi_{\mathcal{C}}^{(k)}
$$

are neighborhoods of the collision set with $\Delta=\bigcap_{k>0} \Xi^{(k)}$.

Not only is the boundary $\partial \Xi^{(k)}$ of $\Xi^{(k)} \subseteq M$ contained in $\widehat{M}$, but there is a lower bound for $q_{\text {min }}$ defined in (2.14):

Lemma 3.6 (Minimal Particle Distance) With $J^{(k)}$ from Definition 3.4,

$$
\partial \Xi^{(k)}=\left\{q \in \Xi^{(k)} \mid J^{(k)}(q)=J(q)+k \delta^{n}\right\} .
$$

There is a $C_{2}>0$ with

$$
q_{\min } \geq C_{2} \sqrt{k} \quad\left(q \in \partial \Xi^{(k)}\right) .
$$

Proof: As $J=J_{\mathcal{C}_{\min }}^{E}, J^{(k)} \geq J+k \delta^{n}$ by Definition 3.4. Thus $q \in M$ satisfies $J^{(k)}(q)=J(q)+k \delta^{n}$ iff $q \in \Xi_{\mathcal{C}_{\text {min }}}^{(k)}$.

If additionally $q \in \Xi^{(k)}$, then there is a $\mathcal{C} \in \mathcal{P}_{0}(N)$ with $q \in \Xi_{\mathcal{C}}^{(k)}$, too. Since $\mathcal{C} \neq \mathcal{C}_{\text {min }}$, we conclude that $q \in \partial \Xi^{(k)}$.

Conversely $\partial \Xi^{(k)} \subseteq\left\{q \in \Xi^{(k)} \mid J^{(k)}(q)=J(q)+k \delta^{n}\right\}$, since

$$
\partial \Xi^{(k)}=\partial\left(M \backslash \Xi^{(k)}\right)=\partial\left(\operatorname{int}\left(\Xi_{\mathcal{C}_{\text {min }}}^{(k)}\right)\right) .
$$

Let $q \in \partial \Xi^{(k)} \cap \partial \Xi_{\mathcal{C}}^{(k)}$ and indices $i, j \in N$ so that $q_{\min }(q)=\left\|q_{i}-q_{j}\right\|$. 
- If $[i]_{\mathcal{C}} \neq[j]_{\mathcal{C}}$, then it follows from (3.13) and (3.12) that $\left\|q_{i}-q_{j}\right\| \geq C^{E} \sqrt{k} / 2$.

- Otherwise $[i]_{\mathcal{C}}=[j]_{\mathcal{C}}$, but $q \in \Xi_{\mathcal{C}_{\min }}^{(k)}$ so that $[i]_{\mathcal{C}_{\min }} \neq[j]_{\mathcal{C}_{\min }}$. For $q \in \partial \Xi^{(k)}$ in particular $J_{\{i, j\}}^{I} \geq k\left(\delta^{n-1}-\delta^{n}\right)$ for all $i \neq j$, so that $q_{\min } \geq C k^{1 / 2}$.

Later, in (3.18), we will define a sequence of hypersurfaces in configuration space $\widehat{M}$ to which our Poincaré surfaces in $\widehat{P}$ are to project. Therefore we now estimate the Riemannian hypersurface volumes of $\partial \Xi^{(k)}$, intersected with balls

$$
B_{R}:=\left\{q \in M \mid\|q\|_{\mathcal{M}} \leq R\right\},
$$

whose radius $R$ goes to infinity as $k \searrow 0$. In the Euclidean space $\left(M,\langle\cdot, \cdot\rangle_{M}\right)$ (see $(2.1))$, the $(n d-1)$-dimensional Riemannian hypersurface volume element is denoted by $d \mathcal{F}$. To obtain an easy upper bound, we instead estimate the $d \mathcal{F}$-volumes of the cylinders

$$
Z_{\mathcal{C}}^{(k)}:=\left\{q \in M \mid J_{\mathcal{C}}^{I}(q)=\eta_{\mathcal{C}}\right\} \cong \Delta_{\mathcal{C}}^{E} \times\left\{q \in \Delta_{\mathcal{C}}^{I} \mid J_{\mathcal{C}}^{I}(q)=\eta_{\mathcal{C}}\right\} \quad\left(\mathcal{C} \in \mathcal{P}_{0}(N)\right),
$$

intersected with $B_{R}$, with $\eta_{\mathcal{C}}:=k\left(\delta^{|\mathcal{C}|}-\delta^{n}\right)>0 . \quad Z_{\mathcal{C}}^{(k)}$ is diffeomorphic to $\mathbb{R}^{d|\mathcal{C}|} \times S^{d(n-|\mathcal{C}|)-1}$. Notice that, unlike on $\partial \Xi_{\mathcal{C}}^{(k)}$, the potential $V$ may diverge on $Z_{\mathcal{C}}^{(k)}$ and is even undefined on the measure zero set $Z_{\mathcal{C}}^{(k)} \cap \Delta$.

Lemma 3.7 There is a decomposition of the boundary $\partial \Xi^{(k)}$ as the union of

$$
\partial \Xi_{\mathcal{C}}^{(k)} \cap \partial \Xi^{(k)} \subseteq Z_{\mathcal{C}}^{(k)} \quad\left(\mathcal{C} \in \mathcal{P}_{0}(N)\right) .
$$

There exists $C>0$ with

$$
\int_{\partial \Xi^{(k)}} \mathbb{1}_{B_{R}} d \mathcal{F} \leq C k^{(d-1) / 2} R^{d(n-1)} \quad(0<k \leq 1 \leq R) .
$$

Proof: By definition, $\Xi^{(k)}=\left\{q \in M \mid J_{0}^{(k)}(q)=J^{(k)}(q)\right\}$ with

$$
J_{0}^{(k)}: M \rightarrow \mathbb{R} \quad, \quad J_{0}^{(k)}(q):=\max \left\{J_{\mathcal{C}}^{E}(q)+k \delta^{|\mathcal{C}|} \mid \mathcal{C} \in \mathcal{P}_{0}(N)\right\} .
$$

So with $\eta_{\mathcal{C}}=k\left(\delta^{|\mathcal{C}|}-\delta^{n}\right)$, using (3.8),

$$
\partial \Xi^{(k)}=\left\{q \in M \mid \exists \mathcal{C} \in \mathcal{P}_{0}(N): J_{\mathcal{C}}^{I}(q)=\eta_{\mathcal{C}}, \forall \mathcal{D} \in \mathcal{P}_{0}(N): J_{\mathcal{D}}^{I}(q) \geq \eta_{\mathcal{D}}\right\} .
$$

On the other hand, by (3.9),

$$
\partial \Xi_{\mathcal{C}}^{(k)} \subseteq\left\{q \in M \mid J_{\mathcal{C}}^{I}(q) \leq \eta_{\mathcal{C}}\right\} \quad\left(\mathcal{C} \in \mathcal{P}_{0}(N)\right) .
$$


Thus $J_{\mathcal{C}}^{I}(q)=\eta_{\mathcal{C}}$ for $q \in \partial \Xi_{\mathcal{C}}^{(k)} \cap \partial \Xi^{(k)}$, showing (3.16). This implies

$$
\int_{\partial \Xi^{(k)}} \mathbb{1}_{B_{R}} d \mathcal{F}=\sum_{\mathcal{C} \in \mathcal{P}_{0}(N)} \int_{\partial \Xi_{\mathcal{C}}^{(k)} \cap \partial \Xi^{(k)}} \mathbb{1}_{B_{R}} d \mathcal{F} \leq \sum_{\mathcal{C} \in \mathcal{P}_{0}(N)} \int_{Z_{\mathcal{C}}^{(k)}} \mathbb{1}_{B_{R}} d \mathcal{F}
$$

with the cylinders $Z_{\mathcal{C}}^{(k)}$. So by (3.2) and (3.3)

$$
\int_{Z_{\mathcal{C}}^{(k)}} \mathbb{1}_{B_{R}} d \mathcal{F} \leq v_{d|\mathcal{C}|} s_{d(n-|\mathcal{C}|)-1}\left(k\left(\delta^{|\mathcal{C}|}-\delta^{n}\right)\right)^{(d(n-|\mathcal{C}|)-1) / 2} R^{d|\mathcal{C}|},
$$

with the volume $v_{m}$ of the $m$-dimensional unit ball and the surface area $s_{m}$ of the sphere $S^{m}$. The estimate follows, since $\max \left\{|\mathcal{C}| \mid \mathcal{C} \in \mathcal{P}_{0}(N)\right\}=n-1$.

From Lemma 3.7 one concludes that $\lim _{k \searrow 0} \int_{\partial \Xi(k)} 1_{B_{R}} d \mathcal{F}=0$, provided that $R \equiv R(k)=o\left(k^{-x_{\max }}\right)$ with $x_{\max }:=\frac{d-1}{2 d(n-1)}$. Thus our assumption $d \geq 2$ allows for divergence of $R$.

Accordingly, if we set for $x \in\left(0, x_{\max }\right)$

$$
k(m):=4^{-m} \text { and } R(m):=4^{m x} \quad(m \in \mathbb{N})
$$

in

$$
\mathcal{F}_{m}:=\bigcup_{\mathcal{C} \in \mathcal{P}_{0}(N)} \mathcal{F}_{m, \mathcal{C}} \quad \text { with } \quad \mathcal{F}_{m, \mathcal{C}}:=\partial \Xi^{(k(m))} \cap \Xi_{\mathcal{C}}^{(k(m))} \cap B_{R(m)},
$$

then $\int_{\mathcal{F}_{m}} d \mathcal{F}=\mathcal{O}\left(2^{-(d-1)\left(1-x / x_{\max }\right) m}\right) \stackrel{m \rightarrow \infty}{\longrightarrow} 0$.

When we are to include integration over momenta, we will have to restrict $x>0$ further, see the proof of Proposition 4.7.

The significance of that family $\left(\mathcal{F}_{m}\right)_{m \in \mathbb{N}}$ of hypersurfaces is clarified by the following lemma:

Lemma 3.8 The forward configuration space trajectory $t \mapsto q(t, x)$ of any initial condition $x \in$ Coll intersects all but finitely many hypersurfaces $\mathcal{F}_{m}$.

Proof: The trajectory has to enter all neighborhoods $\Xi^{(k(m))}$, see (3.14), of the collision set $\Delta$. On the other hand, by definition (2.15) of Coll, the limit $\lim _{t \nearrow T(x)} q(t, x) \in M$ exists. So for the positive time interval $[0, T(x))$ the trajectory stays in a bounded region of $\widehat{M}$, and, by (3.17), is contained in $B_{R(m)}$ for all $m \geq m_{0}$. The claim follows from Definition (3.18).

\section{Remark 3.9 (Symmetries of Hypersurfaces)}

Later, when we estimate the symplectic volumes of the Poincare surfaces erected over the hypersurfaces $\mathcal{F}_{m, \mathcal{C}} \subseteq M$, we will break down that high-dimensional 
integration. One basic step is the factorization $M=\Delta_{\mathcal{C}}^{E} \oplus \Delta_{\mathcal{C}}^{I}$, and its subfactorizations, see (3.4). The cylinder $Z_{\mathcal{C}}^{(k)}$ defined in (3.15) respects these:

$$
Z_{\mathcal{C}}^{(k(m))}=\Delta_{\mathcal{C}}^{E} \times S_{m, \mathcal{C}} \quad \text { with } \quad S_{m, \mathcal{C}}:=\left\{q \in \Delta_{\mathcal{C}}^{I} \mid J_{\mathcal{C}}^{I}(q)=k(m)\left(\delta^{|\mathcal{C}|}-\delta^{n}\right)\right\} .
$$

So $S_{m, \mathcal{C}}$ is a sphere of dimension $d(n-|\mathcal{C}|)-1$, and by (3.16) and (3.18),

$$
\mathcal{F}_{m, \mathcal{C}} \subseteq \Delta_{\mathcal{C}}^{E} \times S_{m, \mathcal{C}}
$$

A decomposition of the factor $\Delta_{\mathcal{C}}^{E}$, adapted to the potential $V$, will be performed using Jacobi coordinates.

\section{Jacobi Coordinates and Maximal Chains}

We will now refine even further the decomposition (3.18) of $\mathcal{F}_{m}$ into the $\mathcal{F}_{m, \mathcal{C}}$. The reason is that we have to cope with the following problem. One could expect that the configuration space trajectory $t \mapsto q(t, x)$ of initial condition $x \in$ Coll, finally colliding in the set partition $\mathcal{C}:=\mathrm{SP}(x) \in \mathcal{P}_{0}(N)$, should intersect the hypersurfaces $\mathcal{F}_{m, \mathcal{C}}$, for large enough $m \in \mathbb{N}$.

However, this need not be the case, since some tight subcluster of particles could form before collision takes place. In Figure 1 that would correspond to a trajectory entering the region $\Xi_{\mathcal{C}_{\max }}^{(k)}$ through one of the channels. This then could lead to intersections of the trajectory with hypersurfaces $\mathcal{F}_{m, \mathcal{D}}$, with $\mathcal{D} \neq \mathcal{C}$ for all large $m$.

At least we can assure that this can only occur if $\mathcal{D} \preccurlyeq \mathcal{C}$ :

\section{Lemma 3.10 (From Finer to Coarser Partitions)}

Let $x \in$ Coll, $\mathcal{D} \in \mathcal{P}_{0}(N)$ and $\left(m_{i}\right)_{i \in \mathbb{N}},\left(t_{i}\right)_{i \in \mathbb{N}}$ be strictly increasing sequences with $q\left(t_{i}, x\right) \in \mathcal{F}_{m_{i}, \mathcal{D}}$. Then $\mathcal{D} \preccurlyeq \mathrm{SP}(x)$.

Proof: As $\lim _{i \rightarrow \infty} k\left(m_{i}\right)=0, \lim _{i \rightarrow \infty} q\left(t_{i}, x\right) \in \Delta_{\mathcal{D}}^{E}$ so that $\lim _{i \rightarrow \infty} t_{i}=T(x)$. From (3.7) it follows that $\Delta_{\mathcal{D}}^{E}=\dot{U}_{\mathcal{C} \succcurlyeq \mathcal{D}} \Xi_{\mathcal{C}}^{(0)}$. So $\mathcal{D} \preccurlyeq \operatorname{SP}(x)$.

The external momentum $p_{\mathcal{C}}^{E}(t, x)$ has a limit as $t \nearrow T(x)$ if $\mathcal{C}=\operatorname{SP}(x)$. This follows simply, since then for $\mathcal{C}=\left(C_{1}, \ldots, C_{k}\right)$ by definition the particles of the same $C_{i}$ converge to the same point, and these points are different for $C_{i}, C_{j}$ with $i \neq j$.

However, the external momentum $p_{\mathcal{D}}^{E}(t, x)$ may diverge in the limit $t \nearrow T(x)$, if $\operatorname{SP}(x) \varsubsetneqq \mathcal{D}$. Thus when in Section 4 we erect the Poincare surfaces $\mathcal{H}_{m}$ in the energy shell over the $\mathcal{F}_{m}$, we have to make them large enough so that they are hit in spite of this divergence. On the other hand the symplectic volume of the Poincaré surface should go to zero as $m \rightarrow \infty$. 
In order to find a definition of the $\mathcal{H}_{m}$ meeting these requirements, we (measure theoretically) decompose the $\mathcal{F}_{m, \mathcal{C}}$ into subsets, indexed by maximal chains

$$
\mathcal{C}_{1} \nsucceq \cdots \varsubsetneqq \mathcal{C}_{k} \quad \text { from } \mathcal{C}_{1}:=\mathcal{C}_{\max } \text { to } \mathcal{C}_{k}:=\mathcal{C} \text {, with }\left|\mathcal{C}_{\ell}\right|=\ell \text {. }
$$

We denote the set of maximal chains ending at $\mathcal{C}$ by $\operatorname{MC}(\mathcal{C})$.

The maximal chain induces a variant of Jacobi coordinates, not for the positions of the bodies but for the cluster barycenters of $\mathcal{C}$. To define them, we use a double index for the clusters of the set partitions:

$$
\mathcal{C}_{\ell}=\left\{C_{\ell, 1}, \ldots, C_{\ell, \ell}\right\} \quad(\ell=1, \ldots, k=|\mathcal{C}|) .
$$

By Remark 3.3.3 there are uniquely two indices $1 \leq L_{\ell}<R_{\ell} \leq \ell$ and an index $1 \leq U_{\ell} \leq \ell-1$ with

$$
C_{\ell-1, U_{\ell}}=C_{\ell, L_{\ell}} \dot{\cup} C_{\ell, R_{\ell}} \quad(\ell=2, \ldots, k),
$$

whereas all other blocks $C_{\ell, i} \in \mathcal{C}_{\ell}$ equal blocks $C_{\ell-1, \pi_{\ell}(i)} \in \mathcal{C}_{\ell-1}$. This attributes to the maximal chain $K=\left(\mathcal{C}_{1}, \ldots, \mathcal{C}_{k}\right) \in \mathrm{MC}(\mathcal{C})$ the linear isomorphism

$$
Q \equiv Q_{K}=\left(Q_{K, 1}, \ldots, Q_{K, k}\right): \Delta_{\mathcal{C}}^{E} \longrightarrow \bigoplus_{j=1}^{k} \mathbb{R}^{d}
$$

with the Jacobi coordinates

$$
Q_{1}:=q_{N} \equiv q_{C_{1,1}} \quad \text { and } \quad Q_{\ell}:=q_{C_{\ell, L(\ell)}}-q_{C_{\ell, R(\ell)}} \quad(\ell=2, \ldots, k) .
$$

So $Q_{1}$ is the center of mass of all particles, and the other $Q_{\ell}$ are the differences of the barycenters of the two clusters to be merged.

The external configuration space region that we attribute to the maximal chain $K=\left(\mathcal{C}_{1}, \ldots, \mathcal{C}_{k}\right) \in \mathrm{MC}(\mathcal{C})$ is its Jacobi space

$$
\mathcal{J}_{K}:=\left\{q \in \Delta_{\mathcal{C}}^{E} \mid \forall \ell \in\{2, \ldots, k\}:\left\|Q_{K, \ell}\right\| \operatorname{Din}_{\mathcal{D} \in \mathcal{P}^{(\ell-1)}, \mathcal{D} \succcurlyeq \mathcal{C}_{\ell}}\left\|q_{C_{\ell, \tilde{L}(\ell)}}-q_{C_{\ell, \tilde{R}(\ell)}}\right\|\right\} .
$$

Here $\tilde{L}(\ell)$ and $\tilde{R}(\ell)$ index the clusters of $\mathcal{C}_{\ell}$ to be merged in $\mathcal{D}$.

For $n=1$ and $n=2$ particles there is only one maximal chain $K$, and $\mathcal{J}_{K}=\Delta_{\mathcal{C}}^{E}$. For all $n$

$$
\Delta_{\mathcal{C}}^{E}=\bigcup_{K \in \operatorname{MC}(\mathcal{C})} \mathcal{J}_{K} \quad\left(\mathcal{C} \in \mathcal{P}_{0}(N)\right)
$$

since $\mathcal{P}(N)$ is a lattice. Conversely, the $\mathcal{J}_{K}$ are disjoint w.r.t. Lebesgue measure,

$$
\lambda^{d|\mathcal{C}|}\left(\mathcal{J}_{K} \cap \mathcal{J}_{L}\right)=0 \quad(K \neq L \in \operatorname{MC}(\mathcal{C})),
$$




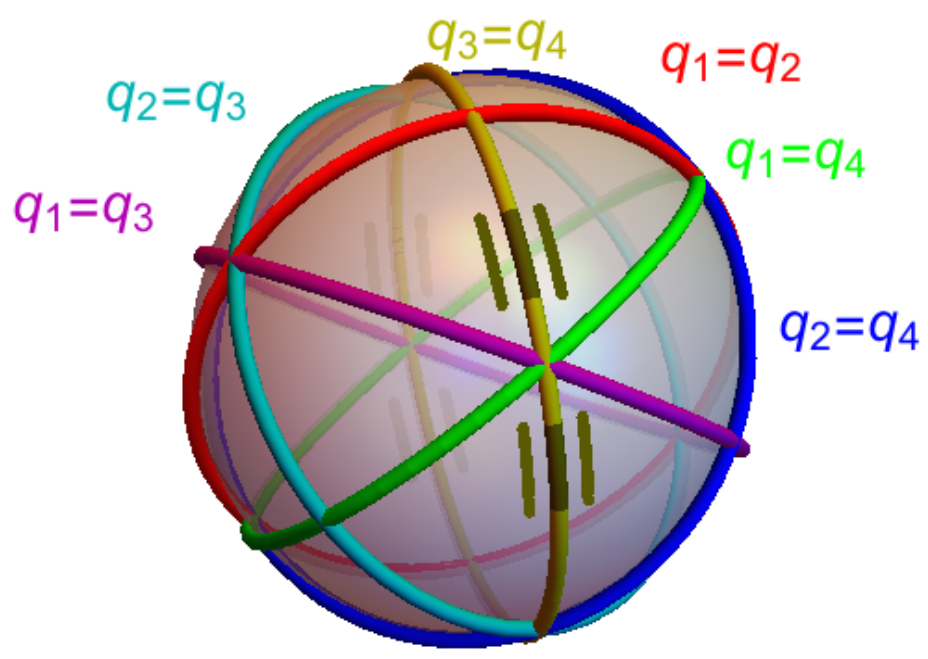

Figure 2: Collision subspaces for $n=4$ particles in $d=1$ dimension, with barycenter at $0 \in \mathbb{R}^{4}$. Shown are only the intersections of these hyperplanes $\left\{q_{i}=q_{j}\right\}$ with the sphere $S^{2}$.

For the maximal chain $K=\left\{\mathcal{C}_{1}, \mathcal{C}_{2}, \mathcal{C}_{3}\right\}$ with $\mathcal{C}=\mathcal{C}_{3}=\{\{1\},\{2\},\{3,4\}\}, \mathcal{C}_{2}=$ $\{\{2\},\{1,3,4\}\}$ and $\mathcal{C}_{1}=\{\{1,2,3,4\}\}=\mathcal{C}_{\max }$, the Jacobi space $\mathcal{J}_{K} \subseteq \Delta_{\mathcal{C}}^{E}$ (that is, in $\left\{q_{3}=q_{4}\right\}$ ) and a hypersurface $\mathcal{F}_{m, K}$ appear in darker color.

since $\left\|Q_{K, \ell}\right\|=\left\|Q_{L, \ell}\right\|$ for $q \in \mathcal{J}_{K} \cap \mathcal{J}_{L}$, but $Q_{K, \ell} \neq Q_{L, \ell}$ for some $\ell$.

Finally, this induces a decomposition of the hypersurfaces (3.18), given by

$$
\mathcal{F}_{m, \mathcal{C}}=\bigcup_{K \in \mathrm{MC}(\mathcal{C})} \mathcal{F}_{m, K} \quad \text { with } \quad \mathcal{F}_{m, K}:=\mathcal{F}_{m, \mathcal{C}} \cap\left(\mathcal{J}_{K} \times \Delta_{\mathcal{C}}^{I}\right) .
$$

The construction is shown in Figure 2. We can locally dominate the inter-cluster potential, using this decomposition:

Lemma 3.11 For all $\mathcal{C} \in \mathcal{P}_{0}(N)$ and maximal chains $K=\left(\mathcal{C}_{1}, \ldots, \mathcal{C}_{k}\right) \in$ $\mathrm{MC}(\mathcal{C})$

$$
J_{\mathcal{C}_{1}}^{E}(q)=m_{N}\left\|Q_{1}\right\|^{2}
$$

and

$$
J_{\mathcal{C}_{\ell}}^{E}(q)-J_{\mathcal{C}_{\ell-1}}^{E}(q)=\frac{m_{C_{\ell, L(\ell)}} m_{C_{\ell, R(\ell)}}}{m_{C_{\ell, L}(\ell)}+m_{C_{\ell, R(\ell)}}}\left\|Q_{\ell}\right\|^{2} \quad(\ell=2, \ldots, k),
$$

with $k=|\mathcal{C}|$. Furthermore, $V=V_{\mathcal{C}}^{E}+V_{\mathcal{C}}^{I}$ with intra- respectively inter-cluster potential

$$
V_{\mathcal{C}}^{E}(q):=\sum_{i \nsim j, i<j} V_{i, j}\left(q_{i}-q_{j}\right) \text { resp. } V_{\mathcal{C}}^{I}(q):=\sum_{i \sim j, i<j} V_{i, j}\left(q_{i}-q_{j}\right) \quad(q \in \widehat{M}),
$$


and for suitable $C_{I}, C_{I I}>0$

$$
\begin{aligned}
\left|V_{\mathcal{C}}^{E}(q)\right| & \leq C_{I}+C_{I I} \sum_{i, j:[i]_{\mathcal{C}} \neq[j]_{\mathcal{C}}}\left\|q_{i}-q_{j}\right\|^{-\alpha} \\
& =C_{I}+\mathcal{O}\left(\left\|Q_{k}\right\|^{-\alpha}\right) \quad\left(q \in \Xi_{\mathcal{C}}^{(k)} \cap\left(\mathcal{J}_{K} \times \Delta_{\mathcal{C}}^{I}\right) \backslash \Delta\right) .
\end{aligned}
$$

\section{Proof:}

As $\mathcal{C}_{1}=\mathcal{C}_{\max }=\{N\}$, the formula for $J_{\mathcal{C}_{1}}^{E}$ follows directly from Definition (3.8). By Remark 3.3.3

$$
J_{\mathcal{C}_{\ell}}^{E}(q)-J_{\mathcal{C}_{\ell-1}}^{E}(q)=\frac{m_{C_{\ell, L(\ell)}} m_{C_{\ell, R(\ell)}}}{m_{C_{\ell-1, U_{\ell}}}}\left\|q_{C_{\ell, L(\ell)}}-q_{C_{\ell, R(\ell)}}\right\|^{2},
$$

and $m_{C_{\ell-1, U_{\ell}}}=m_{C_{\ell, L(\ell)}}+m_{C_{\ell, R(\ell)}}$.

By admissibility, the first inequality of (3.26) is true:

- Definition 2.1 states that $\lim _{\|q\| \rightarrow \infty} V_{i, j}(q)=0$. So for some $C_{I}^{\prime}>0$ we have $\left|V_{i, j}(q)\right| \leq C_{I}^{\prime}$ if $\|q\| \geq 1$.

- Integrating (2.9) twice along the line between $q$ and $q /\|q\|$, we obtain for some $C_{I I}^{\prime}>0$

$$
\left|V_{i, j}(q)\right| \leq C_{I I}^{\prime}\|q\|^{-\alpha} \quad(\|q\| \leq 1) .
$$

We set $C_{I}:=\left(\begin{array}{l}n \\ 2\end{array}\right) C_{I}^{\prime}$ and $C_{I I}:=\left(\begin{array}{l}n \\ 2\end{array}\right) C_{I I}^{\prime}$.

By (3.13) of Lemma 3.5

$$
\sum_{i, j:[i]_{\mathcal{C}} \neq[j]_{\mathcal{C}}}\left\|q_{i}-q_{j}\right\|^{-\alpha} \leq c_{1} \sum_{C, D \in \mathcal{C}: C \neq D}\left\|q_{C}-q_{D}\right\|^{-\alpha} \quad\left(q \in \Xi_{\mathcal{C}}^{(k)} \backslash \Delta\right)
$$

with $c_{1}:=\left(\begin{array}{l}n \\ 2\end{array}\right) 2^{\alpha}$. The definition (3.23) of Jacobi space $\mathcal{J}_{K}$ then implies that $\left\|q_{C}-q_{D}\right\| \geq\left\|Q_{k}\right\|$, leading to the order estimate in (3.26).

\section{Definition and Symplectic Volume of the Poin- caré Surfaces}

Within an energy surface $\Sigma_{E}$ we will define in (4.2) a family $\left(\mathcal{H}_{m}\right)_{m \in \mathbb{N}}$ of hypersurfaces. Any collision orbit in $\Sigma_{E}$ must intersect almost all of the $\mathcal{H}_{m}$. We will also estimate their symplectic volumes, in Proposition 4.7. To perform that task, we will use adapted coordinates. As the coordinate changes are symplectomorphisms, these preserve the volume. We begin by presenting the first such coordinate change, indexed by a cluster decomposition $\mathcal{C}$. 
Using the natural identification $T^{*} M \cong M \times M^{*}$, the decomposition (3.4) of $M$ expands to a $T^{*} M$-orthogonal decomposition

$$
T^{*} M=T^{*} \Delta_{\mathcal{C}}^{E} \oplus T^{*} \Delta_{\mathcal{C}}^{I}=T^{*} \Delta_{\mathcal{C}}^{E} \oplus \bigoplus_{C \in \mathcal{C}} T^{*}\left(\Delta_{C}^{I}\right)
$$

of phase space. The projections onto the corresponding components are denoted by $\widehat{\Pi}_{\mathcal{C}}^{E}$ respectively $\widehat{\Pi}_{\mathcal{C}}^{I}$.

\section{Remark 4.1 (Understanding the Projections $\widehat{\Pi}_{\mathcal{C}}^{E}$ and $\widehat{\Pi}_{\mathcal{C}}^{I}$ )}

For a subspace $N \subseteq M$ of an $\mathbb{R}$-vector space $M$ there is no natural embedding $T^{*} N \subseteq T^{*} M$, although naturally $T N \subseteq T M$. So (4.1) necessitates a definition. Here, using the musical isomorphism

$$
I: M \rightarrow M^{*} \quad, \quad v \mapsto\langle v, \cdot\rangle_{M}=\langle\mathcal{M} v, \cdot\rangle,
$$

we have

$$
\langle I(v), I(w)\rangle_{M^{*}}=\langle\mathcal{M} v, \mathcal{M} w\rangle_{M^{*}}=\langle v, \mathcal{M} w\rangle=\langle v, w\rangle_{M} \quad(v, w \in M) .
$$

So when we identify $T^{*} \Delta_{\mathcal{C}}^{E}$ with $\Delta_{\mathcal{C}}^{E} \times I\left(\Delta_{\mathcal{C}}^{E}\right) \subseteq T^{*} M$ and similarly $T^{*} \Delta_{\mathcal{C}}^{I}$ with $\Delta_{\mathcal{C}}^{I} \times I\left(\Delta_{\mathcal{C}}^{I}\right) \subseteq T^{*} M$, these subspaces are $\langle\cdot, \cdot\rangle_{T^{*} M}$-orthogonal, see (2.2).

By this, we indeed did define symplectic coordinates:

Lemma 4.2 For all $\mathcal{C} \in \mathcal{P}(N)$, the vector space isomorphism

$$
\left(\widehat{\Pi}_{\mathcal{C}}^{E}, \widehat{\Pi}_{\mathcal{C}}^{I}\right): T^{*} M \rightarrow T^{*} \Delta_{\mathcal{C}}^{E} \oplus \bigoplus_{C \in \mathcal{C}} T^{*}\left(\Delta_{C}^{I}\right)
$$

is symplectic w.r.t. to the canonical symplectic forms on the respective cotangent bundles.

Similarly, for all $\mathcal{C} \in \mathcal{P}_{0}(N)$ and maximal chains $K=\left(\mathcal{C}_{1}, \ldots, \mathcal{C}_{k}\right) \in \mathrm{MC}(\mathcal{C})$ the Jacobi map $\mathrm{JM}_{K}$ of phase spaces is symplectic:

\section{Lemma 4.3 (Jacobi Map)}

The inverse cotangent lift of the Jacobi transformation (3.22) has the form

$$
\mathrm{JM}_{K}:=\left(Q_{K}^{*}\right)^{-1}: T^{*} \Delta_{\mathcal{C}}^{E} \rightarrow T^{*}\left(\oplus_{j=1}^{k} \mathbb{R}^{d}\right) \quad, \quad\left(q_{\mathcal{C}}^{E}, p_{\mathcal{C}}^{E}\right) \mapsto(Q, P)
$$

with $(Q, P)=\left(Q_{1}, \ldots, Q_{k}, P_{1}, \ldots, P_{k}\right)$,

$$
Q_{1}=q_{N} \equiv q_{C_{1,1}} \quad, \quad Q_{\ell}=q_{C_{\ell, L_{\ell}}}-q_{C_{\ell, R_{\ell}}} \quad(\ell=2, \ldots, k)
$$

with the notation (3.21), and

$$
P_{1}=p_{N} \equiv p_{C_{1,1}} \quad, \quad P_{\ell}=\frac{m_{C_{\ell, R_{\ell}}} p_{C_{\ell, L_{\ell}}}-m_{C_{\ell, L_{\ell}}} p_{C_{\ell, R_{\ell}}}}{m_{C_{\ell, L_{\ell}}}+m_{C_{\ell, R_{\ell}}}} \quad(\ell=2, \ldots, k) .
$$


Proof: This follows from the facts that

- for $C \subseteq N, C \neq \emptyset$ the same spatial components of the $\mathbb{R}^{d}$-valued functions $q_{C}$ and $p_{C}$ have Poisson bracket one,

- whereas different components of $q_{C}$ and $p_{C}$ have Poisson bracket zero,

- that for $i \in N \backslash C$ the components of $q_{i}$ have Poisson bracket zero with the ones of $p_{C}$,

- and that $q_{C_{\ell-1, U_{\ell}}}=\left(m_{C_{\ell, L_{\ell}}} q_{C_{\ell, L_{\ell}}}+m_{C_{\ell, R_{\ell}}} q_{C_{\ell, R_{\ell}}}\right) / m_{C_{\ell-1, U_{\ell}}}$.

A straightforward calculation shows that kinetic and total energy split into their internal and external parts:

\section{Lemma 4.4 (External and Internal Energies)}

- If we define barycentric and relative kinetic energy by

$$
K_{\mathcal{C}}^{E}:=K \circ \widehat{\Pi}_{\mathcal{C}}^{E} \quad, \quad K_{\mathcal{C}}^{E}(q)=\sum_{C \in \mathbb{C}} K_{C}^{E}(q) \text { with } K_{C}^{E}(q)=\frac{\left\|p_{C}\right\|^{2}}{2 m_{C}}
$$

respectively $K_{\mathcal{C}}^{I}:=K \circ \widehat{\Pi}_{\mathcal{C}}^{I}$, we get $K=K_{\mathcal{C}}^{E}+K_{\mathcal{C}}^{I}$ for all $\mathcal{C} \in \mathcal{P}(N)$.

- Hence, $H=H_{\mathcal{C}}^{E}+H_{\mathcal{C}}^{I}$, with $H_{\mathcal{C}}^{E}=K_{\mathcal{C}}^{E}+V_{\mathcal{C}}^{E}$ resp. $H_{\mathcal{C}}^{I}=K_{\mathcal{C}}^{I}+V_{\mathcal{C}}^{I}$, see (3.25)

In general, however, $H_{\mathcal{C}}^{E}$ and $H_{\mathcal{C}}^{I}$ only Poisson-commute for $\mathcal{C}=\mathcal{C}_{\max }$. Next we erect Poincaré sections $\mathcal{H}_{m} \subseteq \Sigma_{E}$ over $\mathcal{F}_{m}$ :

$$
\mathcal{H}_{m}:=\bigcup_{\mathcal{C} \in \mathcal{P}_{0}(N)} \mathcal{H}_{m, \mathcal{C}} \text { with } \quad \mathcal{H}_{m, \mathcal{C}}:=\bigcup_{K \in \mathrm{MC}(\mathcal{C})} \mathcal{H}_{m, K}
$$

and, using the Jacobi map $\mathrm{JM}_{K}$ of Lemma 4.3 and Definition (3.24) of $\mathcal{F}_{m, K}$,

$$
\begin{aligned}
\mathcal{H}_{m, K}:=\left\{(q, p) \in \Sigma_{E} \mid\right. & q \in \mathcal{F}_{m, K},\left\|P_{1}\right\|^{2} \leq 4^{\beta m}, \\
& \left.\forall \ell \in\{2, \ldots, k\}:\left\|P_{\ell}\right\|^{2} \leq 4^{\beta m}\left\|Q_{\ell}\right\|^{-\alpha}\right\} .
\end{aligned}
$$

\section{Remark 4.5 (The Poincaré Sections)}


1. The $\mathcal{H}_{m, K}$ are well-defined, since for all $q \in \mathcal{F}_{m, \mathcal{C}}$ the $Q_{\ell}(\ell \in\{2, \ldots, k\})$ are nonzero, using Lemma 3.11:

$$
J_{\mathcal{C}}^{E}(q)-J_{\mathcal{D}}^{E}(q) \geq 4^{-m}\left(\delta^{|\mathcal{D}|}-\delta^{|\mathcal{C}|}\right)>0 \quad(\mathcal{D} \varsubsetneqq \mathcal{C}) .
$$

2. Notice that the kinetic energy $K_{\mathcal{C}_{\max }}^{E}=\left\|P_{1}\right\|^{2} /\left(2 m_{N}\right)$ of the center of mass is a constant of the motion for $\Phi$.

3. For regular values $E$ of $V$ the $\mathcal{H}_{m, \mathcal{C}}$ are codimension one $\partial$-submanifolds of the submanifold $\Sigma_{E} \subseteq \widehat{P}$. For critical values $E$ of $V$ we could redefine $\widehat{M}$ by omitting all critical points $q \in \widehat{M}$. Since they are covered by rest points $(q, 0) \in \Sigma_{E}$, this does not change the set $\operatorname{Coll}_{E} \subseteq \Sigma_{E}$, but guarantees the submanifold property of the $\mathcal{H}_{m, \mathcal{C}}$.

However, this redefinition of $\widehat{M}$ is not necessary, as we will work with the subsets $\mathcal{H}_{m, K}^{ \pm} \subseteq \mathcal{H}_{m, K}$, see (4.4) below, that are by their definition $\partial$ submanifolds of $\widehat{P}$, see Lemma 4.6 below.

The cylinders $Z_{\mathcal{C}}^{(k)}$ are hypersurfaces of $\widehat{M}$, thus oriented by an orientation of their normal bundle. This is spanned and oriented by the unit vector field

$$
N_{\mathcal{C}}: Z_{\mathcal{C}}^{(k)} \rightarrow T_{Z_{\mathcal{C}}^{(k)} M} \quad, \quad q \mapsto\left(q, \frac{q_{\mathcal{C}}^{I}}{\left\|q_{\mathcal{C}}^{I}\right\|}\right) .
$$

This allows to decompose the Poincaré sections in two mirror symmetric parts (and a remaining set of zero $\Omega_{n d-1}$-volume), setting

$$
\mathcal{H}_{m, K}^{ \pm}:=\left\{(q, p) \in \mathcal{H}_{m, K} \mid \pm p\left(N_{\mathcal{C}}(q)\right)>0\right\} \quad(K \in \mathrm{MC}(\mathcal{C})) .
$$

Correspondingly,

$$
\mathcal{H}_{m}^{ \pm}:=\bigcup_{\mathcal{C} \in \mathcal{P}_{0}(N)} \mathcal{H}_{m, \mathcal{C}}^{ \pm} \quad \text { with } \quad \mathcal{H}_{m, \mathcal{C}}^{ \pm}:=\bigcup_{K \in \mathrm{MC}(\mathcal{C})} \mathcal{H}_{m, K}^{ \pm}
$$

The $\mathcal{H}_{m, K}^{-}$consist of points on trajectories entering the neighborhood $\Xi^{(k(m))}$ of the collision set. Their embeddings are denoted by

$$
\imath_{m, K}^{ \pm}: \mathcal{H}_{m, K}^{ \pm} \rightarrow T^{*} \widehat{M}
$$

Lemma 4.6 The hypersurfaces $\mathcal{H}_{m, K}^{ \pm} \subseteq \Sigma_{E}(m \in \mathbb{N})$ are transverse to the flow. They are symplectic $\partial$-manifolds. Thus (see (2.3))

$$
\Omega:=\Omega_{n d-1}
$$

induces volume forms $\left(\imath_{m, K}^{ \pm}\right)^{*} \Omega$ on the $\mathcal{H}_{m, K}^{ \pm}$, and their volumes are finite. 
Proof: The $\mathcal{H}_{m, K}^{ \pm}$are transverse to the vector field $X_{H}$ generating the flow $\Phi$, since they project to the hypersurface $\mathcal{F}_{m, K}, X_{H}(q, p)=\left(\mathcal{M}^{-1} p,-\nabla V(q)\right)$ and $\left\langle\mathcal{M}^{-1} p, N_{\mathcal{C}}(q)\right\rangle_{M}=p\left(N_{\mathcal{C}}(q)\right) \neq 0$.

Since $p \neq 0$ in (4.4), the $(q, p) \in \mathcal{H}_{m, K}^{ \pm}$are regular points of the Hamiltonian $H$. The transversality property w.r.t. $X_{H}$ shows that $\mathcal{H}_{m, K}^{ \pm}$are $\partial$-manifolds of $\widehat{P}$. It is a standard argument, see e.g. MCDUFF and SALAMON [MS99, Lemma 8.2], that from such a transversality it follows that the submanifold (or $\partial$-manifold) is symplectic.

As the closures $\overline{\mathcal{H}_{m, K}^{ \pm}}$are compact, they have finite symplectic $\Omega$-volume.

In order to estimate that volume, we project it to $T^{*} \mathcal{F}_{m, K}$, by

$$
n_{m, K}^{ \pm}: \mathcal{H}_{m, K}^{ \pm} \rightarrow T^{*} \mathcal{F}_{m, K} \quad, \quad(q, p) \mapsto\left(q, p-p\left(N_{\mathcal{C}}(q)\right) N_{\mathcal{C}}^{b}(q)\right) .
$$

The cotangent bundle $T^{*} \mathcal{F}_{m, K}$ carries the canonical symplectic form $\omega_{\mathcal{F}}$. Similar to (2.3) and (4.7) we denote the symplectic volume on $T^{*} \mathcal{F}_{m, K}$ by

$$
\widetilde{\Omega}:=\frac{(-1)^{\lfloor k / 2\rfloor}}{k !} \omega_{\mathcal{F}}^{\wedge k}, \quad \text { with } k:=n d-1 .
$$

With respect to the embeddings (4.6) one has

$$
\left(\imath_{m, K}^{ \pm}\right)^{*} \omega_{0}=\left(n_{m, K}^{ \pm}\right)^{*} \omega_{\mathcal{F}},
$$

see Theorem C of [FK18].

By reversibility the two Poincaré sections $\mathcal{H}_{m, K}^{-}$and $\mathcal{H}_{m, K}^{+}$have a common image

$$
\widetilde{\mathcal{H}}_{m, K}:=n_{m, \mathcal{C}}^{ \pm}\left(\mathcal{H}_{m, K}^{ \pm}\right) \subseteq T^{*} \mathcal{F}_{m, K}
$$

Equation (4.9) allows to estimate the $\widetilde{\Omega}$-volume of $\widetilde{\mathcal{H}}_{m, K}$ instead of the $\Omega$ volume of $\mathcal{H}_{m, K}^{ \pm}$. To do this, we use the symplectic Jacobi map $\mathrm{JM}_{K}$ of Lemma 4.3 to present $\widetilde{\Omega}$ in a form adapted to $\widetilde{\mathcal{H}}_{m, K}$.

\section{Proposition 4.7}

By choosing the constants $x>0$ in (3.17) and $\beta>0$ in (4.3) small enough,

$$
\lim _{m \rightarrow \infty} \int_{\mathcal{H}_{m, K}^{ \pm}} \Omega=0 \quad\left(\mathcal{C} \in \mathcal{P}_{0}(N), K \in \mathrm{MC}(\mathcal{C})\right)
$$

for $d \geq 2$ dimensions. So then $\lim _{m \rightarrow \infty} \int_{\mathcal{H}_{m}^{ \pm}} \Omega=0$.

\section{Proof:}

1. By (4.9) and (4.10), $\int_{\mathcal{H}_{m, K}^{ \pm}} \Omega=\int_{\widetilde{\mathcal{H}}_{m, K}} \widetilde{\Omega}$. 
2. Since $q_{\min }(q) \geq C_{2} \sqrt{k}$ for $q \in \partial \Xi^{(k)}$ (Lemma 3.6), and by (3.16),

$$
\widetilde{\mathcal{H}}_{m, K} \subseteq \widehat{\mathcal{H}}_{m, K} \quad \text { and thus } \quad \int_{\widetilde{\mathcal{H}}_{m, K}} \widetilde{\Omega} \leq \int_{\widehat{\mathcal{H}}_{m, K}} \widetilde{\Omega}
$$

with, see (3.19),

$$
\begin{aligned}
\widehat{\mathcal{H}}_{m, K}:= & \left\{(q, p) \in T^{*}\left(\left(\Delta_{\mathcal{C}}^{E} \cap B_{R(m)}\right) \times S_{m, \mathcal{C}}\right) \mid\left\|P_{1}\right\|^{2} \leq 4^{\beta m},\right. \\
& \left.\forall \ell \in\{2, \ldots,|\mathcal{C}|\}:\left\|P_{\ell}\right\|^{2} \leq 4^{\beta m}\left\|Q_{\ell}\right\|^{-\alpha}, K_{\mathcal{C}}^{I} \leq E+c k(m)^{-\alpha / 2}\right\} .
\end{aligned}
$$

This can be seen by comparing with the definition (4.3) of $\mathcal{H}_{m, K}$ and by noting that the projection (4.8) of $\mathcal{H}_{m, K}$ to $\widetilde{\mathcal{H}}_{m, K}$ does not change the Jacobi coordinates. Here $c:=\left(\begin{array}{l}n \\ 2\end{array}\right) C_{V} C_{2}^{-\alpha}$ with $C_{V}$ from (2.11).

3. In Corollary 6.3 of [FK18] the following problem of integration, similar to the present one, is considered. It is assumed that a hypersurface $\mathcal{F} \subseteq M_{1} \times M_{2}$ of the configuration manifold has the property that both families

$$
\mathcal{F}_{1}^{q_{2}}:=\left\{q_{1} \in M_{1} \mid\left(q_{1}, q_{2}\right) \in \mathcal{F}\right\} \quad\left(q_{2} \in M_{2}\right)
$$

and

$$
\mathcal{F}_{2}^{q_{1}}:=\left\{q_{2} \in M_{2} \mid\left(q_{1}, q_{2}\right) \in \mathcal{F}\right\} \quad\left(q_{1} \in M_{1}\right)
$$

consist of hypersurfaces of $M_{1}$ respectively of $M_{2}$. Then for a classical mechanical system with Hamiltonian $H(q, p)=T_{1}\left(q, p_{1}\right)+T_{2}\left(q, p_{2}\right)+V\left(q_{1}, q_{2}\right)$ symplectic volume of a codimension two surface of phase space $T^{*}\left(M_{1} \times M_{2}\right)$, is given by the sum of two integrals, involving $\mathcal{F}_{1}^{q_{2}}$ respectively $\mathcal{F}_{2}^{q_{1}}$.

In the present setting $M_{1}=\Delta_{\mathcal{C}}^{E} \cap B_{R(m)}$ and $M_{2}=\Delta_{\mathcal{C}}^{I}$. The hypersurface $\mathcal{F}$ projects to the sphere $S_{m, \mathcal{C}} \subseteq M_{2}$, so that the conditions of Corollary 6.3 are violated, but in a way that the $\mathcal{F}_{1}^{q_{2}}$ integral vanishes anyhow, and it is enough to treat the $\mathcal{F}_{2}^{q_{1}}$ integral, which we will do below.

4. One advantage of estimating the volume of $\widehat{\mathcal{H}}_{m, K}$ instead of the one of $\widetilde{\mathcal{H}}_{m, K}$ is that the former is defined only by using absolute values of the internal variables. Concerning the external Jacobi variables, we denote their radii by $r_{\ell}:=\left\|Q_{\ell}\right\|$. Since $\widehat{\mathcal{H}}_{m, K}$ is invariant with respect to rotations of the 
corresponding vectors, the integration is reduced to

$$
\begin{aligned}
\int_{\widehat{\mathcal{H}}_{m, K}} \widetilde{\Omega} \leq & \int_{B(R(m))} d Q_{1} \int_{B\left(2^{\beta m}\right)} d P_{1} \prod_{\ell=2}^{|\mathcal{C}|} \int_{B(2 R(m))} \int_{B\left(2^{\beta m}\left\|Q_{l}\right\|^{-\alpha / 2}\right)} d P_{\ell} d Q_{\ell} \\
& \times v_{d(n-|\mathcal{C}|-1)} \int_{S_{m, \mathcal{C}}}\left(E+c k(m)^{-\alpha / 2}\right)^{(d(n-|\mathcal{C}|)-1) / 2} d S_{m, \mathcal{C}} \\
\leq & c_{1} 2^{d \beta m} R(m)^{d} \prod_{\ell=2}^{|\mathcal{C}|}\left(2^{d \beta m} \int_{\mathbb{R}^{d}} \mathbb{1}_{B(R(m))}\left(Q_{\ell}\right) v_{d}\left\|Q_{\ell}\right\|^{-d \alpha / 2} d Q_{\ell}\right) \\
& \times\left(\left(E+c k(m)^{-\alpha / 2}\right)\right)^{(d(n-|\mathcal{C}|)-1) / 2} \int_{S_{m, \mathcal{C}}} d S_{m, \mathcal{C}} \\
= & c_{2} 2^{d|\mathcal{C}| \beta m} R(m)^{d} \prod_{\ell=2}^{|\mathcal{C}|}\left(s_{d-1} v_{d} \int_{0}^{R(m)}\left(r_{\ell}\right)^{(d-1)(1-\alpha / 2)-\alpha / 2} d r_{\ell}\right) \\
& \times\left(\left(E+c k(m)^{-\alpha / 2}\right)\right)^{(d(n-|\mathcal{C}|)-1) / 2} k(m)^{(d(n-|\mathcal{C}|)-1) / 2} \\
= & c_{3} 2^{d|\mathcal{C}| \beta m} R(m)^{d((1-\alpha / 2)|\mathcal{C}|+\alpha / 2)} \\
& \times\left(c_{4}(E)\right)^{(d(n-|\mathcal{C}|)-1) / 2} k(m)^{(1-\alpha / 2)(d(n-|\mathcal{C}|)-1) / 2}=: \operatorname{Int}(m),
\end{aligned}
$$

again with the volume $v_{m}$ of the $m$-dimensional unit ball, the surface area $s_{m}$ of the sphere $S^{m}$, with

$$
\begin{aligned}
c_{1} & :=2^{d(|\mathcal{C}|-1)} v_{d}^{2} v_{d(n-|\mathcal{C}|)-1} \quad, \quad c_{2}:=c_{1} s_{d(n-|\mathcal{C}|)-1}\left(\delta^{|\mathcal{C}|}-\delta^{n}\right)^{(d(n-|\mathcal{C}|)-1) / 2}, \\
c_{3} & :=c_{2}\left(\frac{s_{d-1} v_{d}}{d(1-\alpha / 2)}\right)^{|\mathcal{C}|-1} \text { and } c_{4}(E):=c+|E| k(1)^{\alpha / 2} .
\end{aligned}
$$

We note that $k(m)$ has an exponent that decreases in $|\mathcal{C}|$, whereas the exponent of $R(m)$ and the exponent linear in $\beta$ increase. Here $\mathcal{C} \in \mathcal{P}_{0}(N)$ so that $|\mathcal{C}| \leq n-1$. So when we substitute $k(m)=4^{-m}$ and $R(m):=4^{m x}$ with $x \in\left(0, x_{\max }\right)$ from (3.17), we obtain a $\mathcal{C}$-independent estimate when we choose in all three cases

$$
|\mathcal{C}|=n-1 \text {. }
$$

With $c_{5}(E):=c_{3}\left(c_{4}(E)\right)^{(d(n-|\mathcal{C}|)-1) / 2}$, we get

$$
\operatorname{Int}(m) \leq c_{5}(E) 2^{m \beta d(n-1)} 2^{m x d((1-\alpha / 2)(n-1)+\alpha / 2)} 2^{-m(1-\alpha / 2)(d-1)} .
$$

For $\beta>0$ and $x>0$ both small enough

$$
\lim _{m \rightarrow \infty} \operatorname{Int}(m)=0
$$

since by assumption $\alpha<2$ and $d \geq 2$. 


\section{Remark 4.8 (Binary and Multiple Collisions)}

Note that $|\mathcal{C}|=n-1$ in (4.11) exactly corresponds to the case of a binary collision, that is, there is exactly one non-trivial cluster consisting of two particles. The fact that this case corresponds to the 'worst-case'-scenario regarding the size of the integral $\operatorname{Int}(m)$ is consistent with the heuristic consideration, based on the dimension (3.2) of collision subspaces $\Delta_{\mathcal{C}}^{E}$ in configuration space, that binary collisions should be the "most probable" ones.

Notice, however that the quotient of the phase space integrals of binary and of multiple collisions becomes independent of the parameter $m$ as $\alpha \nearrow 2$. Then the above intuition becomes wrong.

\section{Time Integral of Kinetic Energy}

Below in Proposition 5.6 we will prove finiteness of the time integral of kinetic energy. Our proof method can be considered as based on the one for Chakerian's packing theorem. We present here only its most basic version.

\section{Theorem 5.1 (Chakerian's Packing Theorem [Su])}

For a regular curve $c \in C^{2}\left(\left[s_{0}, s_{1}\right], \mathbb{R}^{k} \backslash\{0\}\right)$, parameterized by arc length, and thus of length $L(c)=s_{1}-s_{0}$ and with curvature $\kappa(s)=\|\ddot{c}(s)\|$,

$$
L(c) \leq\left\|c\left(s_{1}\right)-c\left(s_{0}\right)\right\|+\int_{s_{0}}^{s_{1}}\|c(s)\| \kappa(s) d s .
$$

Proof: $L(c)=\int_{s_{0}}^{s_{1}}\langle\dot{c}(s), \dot{c}(s)\rangle d s=\left.\langle c(s), \dot{c}(s)\rangle\right|_{s_{0}} ^{s_{1}}-\int_{s_{0}}^{s_{1}}\langle c(s), \ddot{c}(s)\rangle d s$.

Remark 5.2 (Chakerian's Packing Theorem) One consequence is that inside a unit ball $L(c) \leq 2+\int_{s_{0}}^{s_{1}} \kappa(s) d s$, so in order to be long, the curve must have mean curvature larger than $1-\varepsilon$. In spite of the simplicity of its proof, the theorem has many interesting implications, see Sullivan [Su, Section 6].

We first transfer this in Lemma 5.3 to motion in admissible potentials $V \in$ $C^{2}\left(\mathbb{R}^{d} \backslash\{0\}, \mathbb{R}\right)$ with $\nabla V(q)=\mathcal{O}\left(\|q\|^{-\alpha-1}\right)(q \rightarrow 0)$ for some $\alpha \in(0,2)$ and, say $\lim _{\|q\| \rightarrow \infty} V(q)=0$. So we consider the Hamiltonian flow line $t \mapsto(q(t), p(t))$ with initial condition $x \in$ Coll on phase space $P:=T^{*}\left(\mathbb{R}^{k} \backslash\{0\}\right)$ for

$$
H \in C^{2}(P, \mathbb{R}) \quad, \quad H(q, p)=\frac{1}{2}\|p\|^{2}+V(q) .
$$

We additionally assume that for some $C_{V}>0$

$$
\langle q, \nabla V(q)\rangle \leq C_{V}+\alpha V_{-}(q) \quad(\|q\| \leq 1),
$$


with $V_{-}(q):=\max (-V(q), 0){ }^{2}$ Here we can simply set

$$
\text { Coll : }=\left\{x \in P \mid \lim _{t \nearrow T(x)} q(t, x)=0\right\} .
$$

\section{Lemma 5.3 (Integral of Kinetic Energy - Potential Scattering)}

Under this assumption the collision trajectories have a finite integral of kinetic energy:

$$
\int_{0}^{T(x)}\|p(t, x)\|^{2} d t<\infty \quad(x \in \text { Coll }) .
$$

\section{Proof:}

For $\tau \in(0, T(x))$ let $\mathcal{K}(\tau):=\int_{0}^{\tau}\|p(t)\|^{2} d t=\int_{0}^{\tau}\|\dot{q}(t)\|^{2} d t$. For $E:=H(x)$ the curve is reparameterized to unit speed by the diffeomorphism to its image $s(t):=$ $\int_{0}^{t} \sqrt{2\left(E-V\left(q\left(t^{\prime}\right)\right)\right)} d t^{\prime}$. The image equals $(0, S)$, with $S:=\lim _{t \rightarrow T(x)} s(t) \in$ $(0, \infty]$. So assuming without loss of generality that $\|q(t)\| \leq 1$ for $t \in[0, T(x))$, with the Heaviside function $\theta$,

$$
\begin{aligned}
\mathcal{K}(\tau) & =\int_{0}^{\tau}\langle\dot{q}(t), \dot{q}(t)\rangle d t=\left.\langle q(t), \dot{q}(t)\rangle\right|_{0} ^{\tau}-\int_{0}^{\tau}\langle q(t), \ddot{q}(t)\rangle d t \\
= & \left.\langle q(t), \dot{q}(t)\rangle\right|_{0} ^{\tau}+\int_{0}^{\tau}\langle q(t), \nabla V(q(t))\rangle d t \\
\leq & \left.\langle q(t), \dot{q}(t)\rangle\right|_{0} ^{\tau}+\int_{0}^{\tau} \theta\left(V(q(t)) C_{V} d t\right. \\
& \quad+\int_{0}^{\tau} \theta\left(-V(q(t)) \frac{C_{V}-\alpha V(q(t))}{2(E-V(q(t)))}\langle\dot{q}(t), \dot{q}(t)\rangle d t\right. \\
\leq & \left.\langle q(t), \dot{q}(t)\rangle\right|_{0} ^{\tau}+\left(C_{V}-\alpha E\right) \tau+\int_{0}^{\tau} \theta(-V(q(t))) \frac{\alpha}{2}\langle\dot{q}(t), \dot{q}(t)\rangle d t \\
\leq & \left.\langle q(t), \dot{q}(t)\rangle\right|_{0} ^{\tau}+\left(C_{V}-\alpha E\right) \tau+\int_{0}^{\tau} \frac{\alpha}{2}\langle\dot{q}(t), \dot{q}(t)\rangle d t .
\end{aligned}
$$

The function $\tau \mapsto\langle q(\tau), \dot{q}(\tau)\rangle$ is bounded on $[0, T(x))$, since $\tau \mapsto\|q(\tau)\|$ is bounded and

$$
\lim _{\|q\| \searrow 0}\|q\| \sqrt{E-V(q)} \leq \lim _{\|q\| \searrow 0}\|q\| \sqrt{E+C\|q\|^{-\alpha}} \leq \lim _{\|q\| \searrow 0}\|q\|(2 C\|q\|)^{-\alpha / 2}=0 .
$$

So

$\lim _{\tau \nearrow T(x)} \mathcal{K}(\tau) \leq(1-\alpha / 2)^{-1}\left(\left.\langle q(t), \dot{q}(t)\rangle\right|_{0} ^{T(x)}+\left(C_{V}-\alpha E\right) \tau\right)<\infty$.

${ }^{2}$ This condition on $V$ corresponds to (2.11) for $n=2$ particles, which is in that case more general than (2.10) in Definition 2.1. 


\section{Remark 5.4}

1. In particular, by Cauchy's inequality, the length $\lim _{\tau \rightarrow T(x)} \int_{0}^{\tau}\|\dot{q}(t)\| d t$ of the collision curve is finite.

2. Condition (5.1) is met (with $\alpha \in[\hat{\alpha}, 2)$ and $C_{V}:=0$ ) by all potentials of the form

$$
V(q)=Z\|q\|^{-\hat{\alpha}} \quad \text { with } \quad \hat{\alpha} \in(0,2) \text { and } Z \in \mathbb{R} .
$$

3. Condition (5.1) alone, without the assumption $\nabla V(q)=\mathcal{O}\left(\|q\|^{-\alpha-1}\right)$, implies that $V_{-}(q)=\mathcal{O}\left(\|q\|^{-\alpha}\right)$.

This follows by using the solution $W(r)=\left(C_{V} / \alpha+W(1)\right) r^{-\alpha}-C_{V} / \alpha$ of the differential equation $r W^{\prime}(r)=-C_{V}-\alpha W(r)$ and by integrating radially.

But (5.1) is not implied by this estimate.

4. As the following example shows, the estimate $V_{-}(q)=\mathcal{O}\left(\|q\|^{-\alpha}\right)$ is not sufficient to prove the statement of Lemma 5.3, even when sharpened to an estimate of the form $\|\nabla V(q)\|=\mathcal{O}\left(\|q\|^{-\alpha-1}\right)$ for some $\alpha \in(0,2)$.

\section{Example 5.5 (Moving on a Spiral: Optimality of Condition (5.1))}

The construction of the potential is based on the lituus spiral

$$
c \in C^{\infty}\left([1, \infty), \mathbb{R}^{2}\right) \quad, \quad c(s)=(\cos (s), \sin (s)) / s^{2} .
$$

It converges to $\lim _{s \rightarrow+\infty} c(s)=0$, and its speed equals $\left\|c^{\prime}(s)\right\|=\sqrt{4+s^{2}} / s^{3}$, so that it is of finite length. The cosine of the angle between $c(s)$ and $c^{\prime}(s)$ equals $-2 / \sqrt{4+s^{2}}$; in particular the angle converges to $\pi / 2$.

We find a more suitable (time) parameterization $t \mapsto \tilde{c}(t):=c(s(t))$ by the assumptions, justified by 1.) and 2.) below,

$$
\left\|\frac{d \tilde{c}(t)}{d t}\right\|=\sqrt{2} s(t) \quad \text { and } \quad s(0)=1 .
$$

Thus $\frac{d t}{d s}=\left\|c^{\prime}(s t)\right\| /(\sqrt{2} s(t))=\sqrt{2+s(t)^{2} / 2} / s(t)^{4}$, and

$$
t(s)=\frac{\left(5 \sqrt{5} s-\sqrt{s^{2}+4}\right) s^{2}-4 \sqrt{s^{2}+4}}{12 \sqrt{2} s^{3}} .
$$

An asymptotic solution of the inverse near $s=\infty$ is: $s(t) \sim\left(2^{3 / 2}(T-t)\right)^{-1 / 2}$, with collision time $T:=(5 \sqrt{5}-1) /(12 \sqrt{2})$.

We now sketch how to find a potential $V \in C^{2}\left(\mathbb{R}^{2} \backslash\{0\}, \mathbb{R}\right)$ with the following properties. 


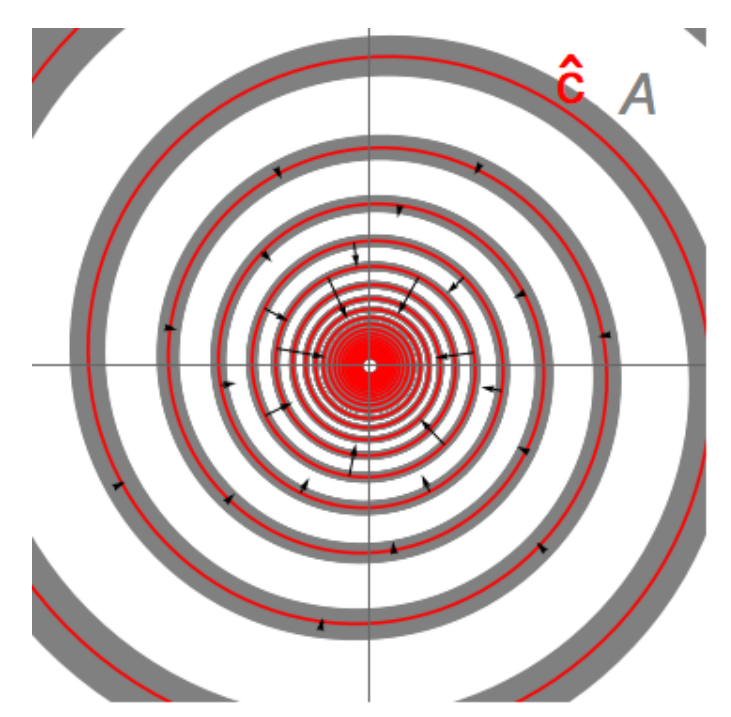

Figure 3: Lituus spiral $\hat{c}$, its neighborhood $A$, and the force field $-\nabla V$ along the spiral

1. Along the curve we set $V(c(s)):=-1 /\|c(s)\|=-s^{2}$, so that for $E:=0$ the speed of a particle in $V$ with holonomic condition set by $c$ equals $\sqrt{2} s$.

2. In order to let the trajectory $\tilde{c}$ of the Hamiltonian $H(q, p)=\frac{1}{2}\|p\|^{2}+V(q)$ move on the image $\hat{c}$ of $c$, the component of $\nabla V(\tilde{c}(t))$ perpendicular to $c^{\prime}(s(t))$ must be equal to the corresponding component of the acceleration $d^{2} \tilde{c} / d t^{2}(t)$. That component is of size $\frac{2 s^{5}\left(s^{2}+2\right)}{\left(s^{2}+4\right)^{3 / 2}}$.

These data determine $V$ and its gradient along $c$, see Figure 3. As the component of $\nabla V$ perpendicular to $c^{\prime}$ is non-vanishing, one can smoothly extend $V$ to a small closed neighborhood $A \subseteq \mathbb{R}^{2} \backslash\{0\}$ of $\hat{c}$. Using Tietze's extension theorem, one finds a continuous extension $\bar{V} \in C\left(\mathbb{R}^{2} \backslash\{0\}, \mathbb{R}\right)$. Applying the theorem locally, $\bar{V}$ even preserves the property $\bar{V}(q)=\mathcal{O}\left(\|q\|^{-1}\right)$.

Then this function is smoothened on small neighborhoods of $\left(\mathbb{R}^{2} \backslash\{0\}\right) \backslash A$, not containing $\hat{c}$. This can be done by convolution with a smooth function, whose support has radius $\|q\|^{-2}$, thus being smaller than the distance of neighboring segments of the lituus spiral.

The function $V$ is then still of order $V(q)=\mathcal{O}\left(\|q\|^{-1}\right)$, like the Kepler potential. $\|\nabla V(c(s))\|$ is asymptotic to $2 s^{4}=2\|c(s)\|^{-2}$. So by the above remark on the angle between $c(s)$ and $c^{\prime}(s)$,

$$
\langle c(s), \nabla V(c(s))\rangle \sim 2 V(c(s)),
$$

which means that the assumption (5.1) of Lemma 5.3 just fails to be true. 
The time integral of kinetic energy, which was finite in Lemma 5.3 is now infinite:

$\int_{0}^{T}\left\|\frac{d \tilde{c}(s(t))}{d t}\right\|^{2} d t=\int_{0}^{T} s^{2} d t=\int_{1}^{\infty} s\left\|c^{\prime}(s)\right\| d s=\int_{1}^{\infty} \sqrt{4+s^{2}} / s^{2} d s=\infty$.

Example 5.5 provides the justification for condition (2.11) in our definition of admissible potentials.

Proposition 5.6 (Integral of Kinetic Energy - $n$-Body Scattering)

For admissible potentials (see Definition 2.1)

$$
\int_{0}^{T(x)} K(p(t, x)) d t<\infty \quad(x \in \text { Coll }) .
$$

\section{Proof:}

- The proof is similar to the one of Lemma 5.3. As the kinetic energy $K_{\mathcal{C}_{\min }}^{E}$ of the barycenter is a constant of the motion, $\mathcal{K}(\tau):=\int_{0}^{\tau} K_{\mathcal{C}_{\text {min }}}^{I}(p(t)) d t=$

$$
\begin{aligned}
= & \int_{0}^{\tau} \sum_{i \in N} \frac{m_{i}\left\|\dot{q}_{i}\right\|^{2}}{2} d t-\frac{\left\|\sum_{i \in N} p_{i}(0)\right\|^{2}}{2 m_{N}} \tau=\frac{1}{2 m_{N}} \sum_{i<j \in N} m_{i} m_{j} \int_{0}^{\tau}\left\|\dot{q}_{i}-\dot{q}_{j}\right\|^{2} d t \\
= & \left.\sum_{i<j \in N} \frac{m_{i} m_{j}}{2 m_{N}}\left\langle q_{i}(t)-q_{j}(t), \dot{q}_{i}(t)-\dot{q}_{j}(t)\right\rangle\right|_{0} ^{\tau} \\
& \quad-\sum_{i<j \in N} \int_{0}^{\tau} \frac{m_{i} m_{j}}{2 m_{N}}\left\langle q_{i}(t)-q_{j}(t), \ddot{q}_{i}(t)-\ddot{q}_{j}(t)\right\rangle d t
\end{aligned}
$$

We treat the terms (5.3) and (5.4) separately.

- (5.3) is uniformly bounded for $\tau \in[0, T(x))$. To show this, we consider

$$
J_{\mathcal{C}_{\text {min }}}^{I}(q)=\sum_{i<j \in N} \frac{m_{i} m_{j}}{m_{N}}\left\|q_{i}-q_{j}\right\|^{2}
$$

see (3.8). Its time derivative

$$
\frac{d}{d t} J_{\mathcal{C}_{\text {min }}}^{I}(q(t))=2 \sum_{i<j \in N} \frac{m_{i} m_{j}}{m_{N}}\left\langle q_{i}(t)-q_{j}(t), \dot{q}_{i}(t)-\dot{q}_{j}(t)\right\rangle
$$

along the orbit is four times the integrand of (5.3). Its second derivative equals

$$
\frac{d^{2}}{d t^{2}} J_{\mathcal{C}_{\text {min }}}^{I}(q)=2 K_{\mathcal{C}_{\text {min }}}^{I}(p)-\sum_{i<j \in N}\left\langle q_{i}-q_{j}, \nabla V_{i, j}\left(q_{i}-q_{j}\right)\right\rangle .
$$

For both alternative conditions of admissibility, this is bounded below: 
1. Assuming (2.10),

$$
\frac{d^{2}}{d t^{2}} J_{\mathcal{C}_{\text {min }}}^{I}(q) \geq 2 K_{\mathcal{C}_{\text {min }}}^{I}(p)+\alpha \sum_{i<j \in N} \frac{Z_{i, j}}{\left\|q_{i}-q_{j}\right\|^{\alpha}}-\left(\begin{array}{c}
n \\
2
\end{array}\right) C_{V} .
$$

By Remark 2.3 this implies

$$
\frac{d^{2}}{d t^{2}} J_{\mathcal{C}_{\text {min }}}^{I}(q) \geq 2 K_{\mathcal{C}_{\text {min }}}^{I}(p)+\alpha V(q)-C \geq \alpha E^{\prime}-C,
$$

with $E^{\prime}:=E-K_{\mathcal{C}_{\text {min }}}^{E}(p(0))$.

2. Assuming (2.11),

$$
\begin{aligned}
\frac{d^{2}}{d t^{2}} J_{\mathcal{C}_{\text {min }}}^{I}(q) & \geq 2 K_{\mathcal{C}_{\min }^{I}}^{I}(p)-\alpha \sum_{i<j \in N}\left(V_{i, j}\right)_{-}\left(q_{i}-q_{j}\right)-\left(\begin{array}{l}
n \\
2
\end{array}\right) C_{V} \\
& =2 K_{\mathcal{C}_{\text {min }}}^{I}(p)+\alpha \sum_{i<j \in N} \min \left(V_{i, j}\left(q_{i}-q_{j}\right), 0\right)-\left(\begin{array}{l}
n \\
2
\end{array}\right) C_{V} \\
& \geq 2 K_{\mathcal{C}_{\min }}^{I}(p)+\alpha V(q)-\left(\begin{array}{l}
n \\
2
\end{array}\right)\left(C_{V}+\alpha V_{\max }\right) \\
& \geq \alpha E^{\prime}-\left(\begin{array}{l}
n \\
2
\end{array}\right)\left(C_{V}+\alpha V_{\max }\right),
\end{aligned}
$$

which is finite since by definition of admissibility

$$
V_{\max }:=\sup \left\{V_{i, j}(q) \mid i<j \in N, q \in \mathbb{R}^{d} \backslash\{0\}\right\} \in[0, \infty) .
$$

So $\liminf \operatorname{in}_{t} T_{(x)} \frac{d}{d t} J_{\mathcal{C}_{\min }}^{I}(q(t))>-\infty$.

But as $J_{\mathcal{C}_{\text {min }}}^{I}(q)>0$ and $\lim _{t \nearrow T(x)} J_{\mathcal{C}_{\text {min }}}^{I}(q(t))=0$, we get

$$
\limsup _{t \nearrow T(x)} \frac{d}{d t} J_{\mathcal{C}_{\min }}^{I}(q(t))<+\infty
$$

too: Between any $t \in[0, T(x))$ and $T(x)$ there exists a $t^{\prime}$ with $\frac{d}{d t} J_{\mathcal{C}_{\min }}^{I}\left(q\left(t^{\prime}\right)\right) \leq 0$. So for $t^{\prime \prime} \in[0, T(x))$ we have

$$
\frac{d}{d t} J_{\mathcal{C}_{\text {min }}}^{I}\left(q\left(t^{\prime}\right)\right) \leq-\int_{t^{\prime \prime}}^{T(x)} \frac{d^{2}}{d t^{2}} J_{\mathcal{C}_{\text {min }}}^{I}(q(t)) d t \leq-\left(C_{V}+\alpha E^{\prime}\right) T(x) .
$$

- For (5.4),

$$
\begin{aligned}
& -\sum_{i<j \in N} \int_{0}^{\tau} \frac{m_{i} m_{j}}{2 m_{N}}\left\langle q_{i}-q_{j}, \ddot{q}_{i}-\ddot{q}_{j}\right\rangle d t \\
& =\sum_{i<j \in N} \int_{0}^{\tau} \frac{m_{i}+m_{j}}{2 m_{N}}\left\langle q_{i}-q_{j}, \nabla V_{i, j}\left(q_{i}-q_{j}\right)\right\rangle d t \\
& +\sum_{i<j \in N} \int_{0}^{\tau} \sum_{k \in N \backslash\{i, j\}} \frac{\left\langle q_{i}-q_{j}, m_{j} \nabla V_{i, k}\left(q_{i}-q_{k}\right)-m_{i} \nabla V_{j, k}\left(q_{j}-q_{k}\right)\right\rangle}{2 m_{N}} d t \\
& =\frac{1}{2} \sum_{i<j \in N} \int_{0}^{\tau}\left\langle q_{i}-q_{j}, \nabla V_{i, j}\left(q_{i}-q_{j}\right)\right\rangle d t .
\end{aligned}
$$


Without loss of generality we assume $\left\|q_{i}-q_{j}\right\| \leq 1$.

1. Assuming condition (2.10) in the definition of admissibility, (5.6) is estimated by

$$
\begin{aligned}
& \frac{1}{2} \sum_{i<j \in N} \int_{0}^{\tau}\left\langle q_{i}-q_{j}, \nabla V_{i, j}\left(q_{i}-q_{j}\right)\right\rangle d t \leq C_{5} \tau-\frac{\alpha}{2} \sum_{i<j \in N} \int_{0}^{\tau} \frac{Z_{i, j}}{\left\|q_{i}-q_{j}\right\|^{\alpha}} d t \\
& \quad \leq C_{6} \tau+\frac{\alpha}{2} \int_{0}^{\tau}\left[E^{\prime}-V(q)\right] d t=C_{6} \tau+\frac{\alpha}{2} \int_{0}^{\tau} K_{\mathcal{C}_{\min }}^{I}(p) d t
\end{aligned}
$$

with $E^{\prime}:=E-K_{\mathcal{C}_{\min }}^{E}(p(0))$ and $C_{6}:=C_{5}+\frac{\alpha}{2}\left(\left(\begin{array}{l}n \\ 2\end{array}\right) C-E^{\prime}\right), C$ being the constant from Remark 2.3.

2. Similarly, for the alternative condition (2.11) of admissibility,

$$
\begin{aligned}
& \frac{1}{2} \sum_{i<j \in N} \int_{0}^{\tau}\left\langle q_{i}-q_{j}, \nabla V_{i, j}\left(q_{i}-q_{j}\right)\right\rangle d t \leq \frac{1}{2} \sum_{i<j \in N} \int_{0}^{\tau}\left[C_{V}+\alpha\left(V_{i, j}\right)_{-}\left(q_{i}-q_{j}\right)\right] d t \\
& =C_{2} \tau+\frac{1}{2} \int_{0}^{\tau} \alpha\left[\sum_{i<j \in N}\left(V_{i, j}\right)_{-}\left(q_{i}-q_{j}\right)\right] d t \\
& \leq C_{3} \tau+\frac{1}{2} \int_{0}^{\tau} \alpha\left[E^{\prime}-V(q)\right] d t \leq C_{3} \tau+\frac{\alpha}{2} \int_{0}^{\tau} K_{\mathcal{C}_{\min }}^{I}(p) d t,
\end{aligned}
$$

with $C_{V}$ from Definition 2.1, $C_{2}:=\frac{1}{2}\left(\begin{array}{l}n \\ 2\end{array}\right) C_{V}$ and $C_{3}:=C_{2}+\frac{\alpha}{2}\left(\left(\begin{array}{l}n \\ 2\end{array}\right) V_{\max }-E^{\prime}\right)$, see (5.5).

- We arrive at an inequality of the form

$$
(2-\alpha) \mathcal{K}(\tau) \leq C_{4}+C_{3} \tau \quad(\tau \in[0, T(x))) .
$$

Since $\alpha<2$, this shows boundedness of (5.2).

The internal cluster energy of a cluster $C \subseteq N$ equals $H_{C}^{I}(q, p)=K_{C}^{I}(p)+V_{C}^{I}(q)$ with $K_{C}^{I}(p):=\sum_{i \in C} \frac{\left\|p_{i}^{I}\right\|^{2}}{2 m_{i}}$ and $V_{C}^{I}(q):=\sum_{i<j \in C} V_{i, j}(q)$. Its limit value at collision time exists:

Corollary 5.7 (Collision Limits) For $x \in \operatorname{Coll}$ and $\mathcal{C}:=\operatorname{SP}(x)$,

$$
\int_{0}^{T(x)} K_{C}^{I}(p) d t<\infty \quad \text { and } \quad \lim _{t \nearrow T(x)} H_{C}^{I}(q(t), p(t)) \in \mathbb{R} \quad(C \in \mathcal{C}) .
$$

The limit $\lim _{t \nearrow T(x)}\left(q_{\mathcal{C}}^{E}(t), p_{\mathcal{C}}^{E}(t)\right)$ of external cluster coordinates exists.

\section{Proof:}

- By Proposition 5.6 the time integral of the total kinetic energy $K$ is bounded 
for collision orbits. As $K=K_{\mathcal{C}}^{E}+K_{\mathcal{C}}^{I}$ with $K_{\mathcal{C}}^{I}=\sum_{C \in \mathcal{C}} K_{C}^{I}$, see Lemma 4.4, and both external and internal kinetic cluster energies are nonnegative, the first statement follows.

- The time derivative along the orbit equals

$\frac{d}{d t} H_{C}^{I}(q, p)=\sum_{i \in C}\left\langle\dot{q}_{i}^{I}(t), \sum_{k \in N \backslash C}\left(-\nabla V_{i, k}\left(q_{i}-q_{k}\right)+\sum_{j \in C} \frac{m_{j}}{m_{C}} \nabla V_{j, k}\left(q_{j}-q_{k}\right)\right)\right\rangle$.

The square of the first vector valued function $t \mapsto \dot{q}_{i}^{I}(t)$ is integrable, using the first statement. The norm of the second vector valued function is uniformly bounded for $t \in[0, T(x))$. By the Cauchy-Schwarz inequality the second statement follows.

- $\lim _{t \nearrow T(x)} q_{\mathcal{C}}^{E}(t)=\lim _{t \nearrow T(x)} q(t)$ exists by definition of a collision singularity. The limit of $p_{\mathcal{C}}^{E}$ exists, since $\inf _{t \in[0, T(x))}\left\|q_{i}(t)-q_{j}(t)\right\|>0$ if $[i]_{\mathcal{C}} \neq[j]_{\mathcal{C}}$. So $\left\|\dot{p}_{\mathcal{C}}^{E}(t)\right\|$ is bounded above on $[0, T(x))$.

\section{Hitting the Poincaré Sections}

Finally we show that almost every collision orbit hits almost all Poincaré surfaces. There is a natural family of Hamiltonians associated to the Jacobi map of Lemma 4.3. To simplify notation, we consider them for one arbitrary index $\ell \in\{2, \ldots, k\}$ and henceforth omit that index. Then (as in Remark 3.3) we have set partitions $\mathcal{C} \preccurlyeq \mathcal{D}$ with $\ell=|\mathcal{C}|=|\mathcal{D}|+1$, and there is a unique cluster $D_{U} \in \mathcal{D}$ which is the disjoint union $C_{L} \dot{\cup} C_{R}$ of two clusters $C_{L}, C_{R} \in \mathcal{C}$, and the other clusters of $\mathcal{D}$ coincide with the other clusters of $\mathcal{C}$. The difference of the external Hamiltonians from Lemma 4.4 is

$$
\mathfrak{H}:=H_{\mathcal{C}}^{E}-H_{\mathcal{D}}^{E}=K_{\mathcal{C}}^{E}-K_{\mathcal{D}}^{E}+V_{\mathcal{C}}^{E}-V_{\mathcal{D}}^{E} .
$$

Here, with reduced mass $\mu:=\frac{m_{C_{L}} m_{C_{R}}}{m_{D_{U}}}$ and Jacobi coordinates

$$
\begin{gathered}
(Q, P)=\left(q_{C_{L}}-q_{C_{R}}, \mu\left(\frac{P_{C_{L}}}{m_{C_{L}}}-\frac{P_{C_{R}}}{m_{C_{R}}}\right)\right) \in T^{*} \mathbb{R}^{d} \\
K_{\mathcal{C}}^{E}(p)-K_{\mathcal{D}}^{E}(p)=\sum_{C \in \mathcal{C}} \frac{\left\|p_{C}\right\|^{2}}{2 m_{C}}-\sum_{D \in \mathcal{D}} \frac{\left\|p_{D}\right\|^{2}}{2 m_{D}}=\frac{\left\|p_{C_{L}}\right\|^{2}}{2 m_{C_{L}}}+\frac{\left\|p_{C_{R}}\right\|^{2}}{2 m_{C_{R}}}-\frac{\left\|p_{D_{U}}\right\|^{2}}{2 m_{D_{U}}} \\
=\frac{\|P\|^{2}}{2 \mu}
\end{gathered}
$$




$$
\begin{aligned}
V_{\mathcal{C}}^{E}(q)-V_{\mathcal{D}}^{E}(q) & =\sum_{i<j \in N:[i]_{\mathcal{C}} \neq[j]_{\mathcal{C}}} V_{i, j}\left(q_{i}-q_{j}\right)-\sum_{i<j \in N:[i]_{\mathcal{D}} \neq[j]_{\mathcal{D}}} V_{i, j}\left(q_{i}-q_{j}\right) \\
& =\sum_{i \in C_{L}, j \in C_{R}} V_{i, j}\left(q_{i}-q_{j}\right)=\sum_{i \in C_{L}, j \in C_{R}} V_{i, j}\left(Q+\left(q_{\mathcal{C}}^{I}\right)_{i}-\left(q_{\mathcal{C}}^{I}\right)_{j}\right) .
\end{aligned}
$$

We now consider the change of the Jacobi kinetic energy (6.1) along a solution curve by the fundamental theorem of calculus:

$$
\frac{\|P(t)\|^{2}}{2 \mu}=\frac{\|P(0)\|^{2}}{2 \mu}+\int_{0}^{t} \frac{d}{d \tau} \frac{\|P(\tau)\|^{2}}{2 \mu} d \tau .
$$

Using (6.1):

$$
\begin{aligned}
\frac{d}{d t} \frac{\|P\|^{2}}{2 \mu}= & \frac{d}{d t}\left(\frac{\left\|p_{C_{L}}\right\|^{2}}{2 m_{C_{L}}}+\frac{\left\|p_{C_{R}}\right\|^{2}}{2 m_{C_{R}}}-\frac{\left\|p_{D_{U}}\right\|^{2}}{2 m_{D_{U}}}\right) \\
= & \left\langle\dot{q}_{C_{L}}, \dot{p}_{C_{L}}\right\rangle+\left\langle\dot{q}_{C_{R}}, \dot{p}_{C_{R}}\right\rangle-\left\langle\dot{q}_{D_{U}}, \dot{p}_{D_{U}}\right\rangle \\
= & -\sum_{i \in C_{L}, j \in N \backslash C_{L}}\left\langle\dot{q}_{C_{L}}, \nabla V_{i, j}\left(q_{i}-q_{j}\right)\right\rangle-\sum_{i \in C_{R}, j \in N \backslash C_{R}}\left\langle\dot{q}_{C_{R}}, \nabla V_{i, j}\left(q_{i}-q_{j}\right)\right\rangle \\
& +\sum_{i \in D_{U}, j \in N \backslash D_{U}}\left\langle\dot{q}_{D_{U}}, \nabla V_{i, j}\left(q_{i}-q_{j}\right)\right\rangle .
\end{aligned}
$$

The three terms have a similar form. We show explicitly how to estimate the first term:

$$
\begin{aligned}
& -\sum_{i \in C_{L}, j \in N \backslash C_{L}}\left\langle\dot{q}_{C_{L}}, \nabla V_{i, j}\left(q_{i}-q_{j}\right)\right\rangle=-\sum_{C \in \mathcal{C} \backslash\left\{C_{L}\right\}} \sum_{i \in C_{L}, j \in C}\left\langle\dot{q}_{C_{L}}, \nabla V_{i, j}\left(q_{i}-q_{j}\right)\right\rangle \\
& =-\frac{d}{d t} W_{L}(q)+\sum_{C \in \mathcal{C} \backslash\left\{C_{L}\right\}} \sum_{i \in C_{L}, j \in C}\left\langle\dot{q}_{C_{L}}, \nabla V_{i, j}\left(q_{i}^{E}-q_{j}^{E}\right)-\nabla V_{i, j}\left(q_{i}-q_{j}\right)\right\rangle,
\end{aligned}
$$

with

$$
W_{L}(q(t)):=\sum_{C \in \mathcal{C} \backslash\left\{C_{L}\right\}} \sum_{i \in C_{L}, j \in C} V_{i, j}\left(q_{i}^{E}(t)-q_{j}^{E}(t)\right) .
$$

By (2.9) the contribution of the first term in (6.4) to (6.2) is of order

$$
W_{L}(q(0))-W_{L}(q(t))=\mathcal{O}\left(\sum_{C \in \mathcal{C} \backslash\left\{C_{L}\right\}}\left\|q_{C_{L}}(t)-q_{C}(t)\right\|^{-\alpha}\right) \quad(t \in[0, T(x))) .
$$

Up to now we did not pose an assumption about the location of the trajectory in configuration space. This, however will be needed in the proof of the following proposition. 


\section{Proposition 6.1 (Hitting the Poincaré Surfaces)}

For every energy $E \in \mathbb{R}$ and $\sigma_{E}$-almost every initial condition $x \in$ Coll there is an $M(x) \in \mathbb{N}$ so that, with $\mathcal{H}_{m}^{ \pm}$defined in (4.4),

$$
\Phi([0, T(x)), x) \cap \mathcal{H}_{m}^{-} \neq \emptyset \quad(m \geq M(x)) .
$$

\section{Proof:}

According to Lemma 3.8, the forward configuration space trajectory $q([0, T(x)), x)$ intersects all but finitely many hypersurfaces $\mathcal{F}_{m}$. By Lemma 3.1 of [FK18] we can assume that the intersections of the forward configuration space trajectory $q([0, T(x)), x)$ with the hypersurfaces $\mathcal{F}_{m}$ is transversal. By transversality and closedness of $\cup_{m \in \mathbb{N}} \mathcal{F}_{m} \subseteq \widehat{M}$, the set

$$
\left\{t \in[0, T(x)) \mid q(t, x) \in \cup_{m \in \mathbb{N}} \mathcal{F}_{m}\right\}
$$

is discrete in $[0, T(x))$. When we enumerate it in ascending order by $\left(t_{i}\right)_{i \in \mathbb{N}}$, then $\lim _{i \rightarrow \infty} t_{i}=T(x)$. As the $\mathcal{F}_{m}$ are mutually disjoint, the indices $m_{i} \in \mathbb{N}$ are uniquely defined by $q\left(t_{i}, x\right) \in \mathcal{F}_{m_{i}}$.

As $\mathcal{P}_{0}(N)$ is finite, there is at least one $\mathcal{D} \in \mathcal{P}_{0}(N)$ with $q\left(t_{i}, x\right) \in \mathcal{F}_{m_{i}, \mathcal{D}}$ for infinitely many $i \in \mathbb{N}$. As stated in Lemma $3.10, \mathcal{D} \preccurlyeq \operatorname{SP}(x)$ for those $\mathcal{D}$.

In fact, it suffices to show that for any $\mathcal{D} \in \mathcal{P}_{0}(N)$ there is a $I(\mathcal{D}) \in \mathbb{N}$ such that

$$
\Phi\left(t_{i}, x\right) \in \mathcal{H}_{m_{i}, \mathcal{D}}^{-} \quad\left(i \geq I(\mathcal{D}): q\left(t_{i}, x\right) \in \mathcal{F}_{m_{i}, \mathcal{D}}\right) .
$$

Then with $I:=\max _{\mathcal{D} \in \mathcal{P}_{0}(N)} I(\mathcal{D})$ and $M(x):=m_{I}$ the proposition is true.

Obviously we have to prove (6.7) only for those $\mathcal{D} \in \mathcal{P}_{0}(N)$ with an infinite number of collisions with hypersurfaces $\mathcal{F}_{m_{i}, \mathcal{D}}$.

The total momentum $P_{1}$ (see Lemma 4.3) is conserved, so that the first condition in the definition (4.3) of the Poincaré surfaces $\mathcal{H}_{m, K}$ is met for $m$ large.

We first consider the case $\mathcal{D}=\operatorname{SP}(x)$. Then not only the external positions $q_{D}^{E}$ but also the momenta $p_{D}^{E}$ for the clusters $D \in \mathcal{D}$ have collision limits, see Corollary 5.7. So also the Jacobi coordinates $Q_{\ell}$ and $P_{\ell}(\ell \in\{2, \ldots, k\})$ from Lemma 4.3 have limits, and the second condition in (4.3) is met, too for $m$ large. The second case to consider is $\mathcal{D} \supsetneqq \mathrm{SP}(x)$. The norms of the Jacobi momenta $P_{\ell}$ are estimated based on (6.2):

$$
\left\|P_{\ell}(t)\right\|^{2}=\left\|P_{\ell}(0)\right\|^{2}+2 \int_{0}^{t}\left\langle P_{\ell}(\tau), \frac{d}{d \tau} P_{\ell}(\tau)\right\rangle d \tau .
$$

We now consider a segment of the trajectory, with $q_{\mathcal{C}}^{E}(t)$ in the Jacobi space $\mathcal{J}_{K}$. Then (6.6) can be bounded by

$$
W_{L}(q(0))-W_{L}(q(t))=\mathcal{O}\left(\left\|Q_{k}\right\|^{-\alpha}\right),
$$


by (3.26) and the definition (3.23) of $\mathcal{J}_{K}$.

To estimate the second term

$$
\sum_{C \in \mathcal{C} \backslash\left\{C_{L}\right\}} \sum_{i \in C_{L}, j \in C}\left\langle\dot{q}_{C_{L}}, \nabla V_{i, j}\left(q_{i}^{E}-q_{j}^{E}\right)-\nabla V_{i, j}\left(q_{i}-q_{j}\right)\right\rangle
$$

in (6.4), we note that, by assumption (2.9) on the potential

$$
\left\|\nabla V_{i, j}\left(q_{i}^{E}-q_{j}^{E}\right)-\nabla V_{i, j}\left(q_{i}-q_{j}\right)\right\| \leq C\left\|q_{i}^{E}-q_{j}^{E}\right\|^{-\alpha-2} \|\left(\left\|q_{i}^{I}\right\|+\left\|q_{j}^{I}\right\|\right) .
$$

But as we assumed that $[i] \neq[j]$, by Lemma 3.5 the internal cluster coordinates are bounded by

$$
\left\|q_{i}^{I}\right\|+\left\|q_{j}^{I}\right\|=\mathcal{O}\left(\left\|q_{i}^{E}-q_{j}^{E}\right\|\right)
$$

So

$$
\left\|\nabla V_{i, j}\left(q_{i}^{E}-q_{j}^{E}\right)-\nabla V_{i, j}\left(q_{i}-q_{j}\right)\right\| \leq C\left\|q_{i}^{E}-q_{j}^{E}\right\|^{-\alpha-1} .
$$

Proposition 5.6 states that the time integral of kinetic energy is finite. So in particular, using the Cauchy-Schwarz inequality, the length $\int_{0}^{T(x)}\left\|\dot{q}_{C_{L}}(t)\right\| d t$ of the collision curve is finite, i.e., we can reparameterize it by arc length, with a bounded parameter interval. By integrating (6.9), we obtain the estimate $\mathcal{O}\left(\left\|Q_{\ell}(t)\right\|^{-\alpha}\right)$ for (6.8), too.

The other two terms in (6.3) are estimated by the same method.

\section{Proof of Theorem 2.5:}

We adapt Theorem A of [FK18] to the notation of the present article. Then it states under the assumptions

1. that the vector field $X_{H}$, restricted to the energy surface $\Sigma_{E}$, is transverse to the Poincaré surfaces $\mathcal{H}_{m}^{-}$,

2. and that these have finite volume, with $\lim _{m \rightarrow \infty} \int_{\mathcal{H}_{m}} \Omega=0$,

that the Liouville measure

$$
\sigma_{E}\left(\operatorname{Trans}_{E} \cap \operatorname{Wand}_{E}\right)=0,
$$

with

3. $\operatorname{Trans}_{E}:=\left\{x \in \Sigma_{E} \mid \exists m_{0} \in \mathbb{N} \forall m \geq m_{0}: \mathcal{O}^{+}(x) \cap \overline{\mathcal{H}}_{m} \neq \emptyset\right\}$, and

4. the wandering set $\operatorname{Wand}_{E}$ consisting of those $x \in \Sigma_{E}$ which have a neighborhood $U_{x}$ so that for a suitable time $t_{-} \in(0, T(x))$

$$
U_{x} \cap \Phi\left(\left(\left(t_{-}, T(x)\right) \times U_{x}\right) \cap D\right)=\emptyset .
$$


This implies that $\sigma_{E}\left(\mathrm{Coll}_{E}\right)=0$, since:

1. Concerning Condition 1. above, Lemma 4.6 states that the Hamiltonian vector field $X_{H}$ is transverse to all $\mathcal{H}_{m}^{-}$.

2. The $2(n d-1)$-form $\Omega=\Omega_{n d-1}$, defined in (4.7) is invariant under the flow generated by $X_{H}$. As proven in (6.1) of [FK18], $i_{X_{H}} \sigma_{E}$ equals (possibly up to sign) the pullback $\imath_{E}^{*} \Omega$ of that form to the energy surface $\Sigma_{E}$.

So Condition 2. has been shown in Lemma 4.6 and Proposition 4.7.

3. Then Proposition 6.1 states that $\mathrm{Coll}_{E} \subseteq$ Trans.

4. As proven in Lemma 1.2 of [FK18], $\operatorname{Sing}_{E} \subseteq \operatorname{Wand}_{E}$. So by Definition (2.15), Coll $_{E} \subseteq$ Wand $_{E}$, too.

\section{Addenda}

\section{Collisions on the Line}

Up to this point, we have used the assumption $d \geq 2$ only in the proof of Proposition 4.7. So the same reasoning can be applied when considering collision orbits in $d=1$ dimension. Obviously, binary collisions cannot be improbable in this case. This is consistent with the last exponent in (4.12) becoming zero for $d=1$ and $|\mathcal{C}|=n-1$ (which exactly corresponds to a single binary collision). However, if we choose $|\mathcal{C}| \leq n-2$ instead of (4.11), that exponent is negative even for $d=1$. This is the case for every multiple collision configuration, that does not consist of merely one binary. Thus we have also shown:

\section{Theorem 7.1}

In $d=1$ dimension, for any energy $E \in \mathbb{R}$ the set of all non-binary collision orbits (i.e. collisions consisting of a triple or higher order collision, of several collisions occurring simultaneously, or a combination thereof) has Liouville measure zero.

\section{Collisions in Systems with Centers}

In Theorem 2.5 the potential $V$ was assumed to be of the form (2.7). Only minor changes need to be implemented in the presence of fixed centers, that is for potentials of the form

$$
V(q)+\sum_{i \in N, k=1, \ldots, k_{\max }} W_{i, k}\left(q_{i}-c_{k}\right),
$$

with $W_{n, k} \in C^{2}\left(\mathbb{R}^{d} \backslash\left\{c_{k}\right\}, \mathbb{R}\right)$ admissible in the sense of Definition 2.1. 
When defining the coordinates, we have to discriminate whether one of the centers is part of the cluster or not. In the first case, internal cluster coordinates are replaced by the particles' distances to the center, whereas the respective external coordinates are dropped (the estimates on the external coordinates' insignificant contribution to phase space volume vanish into the obvious fact that the centers don't contribute to that at all). Otherwise, the coordinates are defined as before. Then the Poincaré surfaces are also defined as before. The rest is a straightforward calculation along the lines of the previous sections.

\section{References}

[DG97] Jan Dereziński and Christian Gérard: Scattering theory of classical and quantum $N$-particle systems. Texts and Monographs in Physics. Springer-Verlag, Berlin, 1997.

[FK18] Stefan Fleischer and Andreas Knauf: Improbability of Wandering Orbits Passing Through a Sequence of Poincaré Surfaces of Decreasing Size. Unpublished, 2018.

[Kn18] Andreas Knauf: Mathematical Physics: Classical Mechanics. Springer, New York, 2018.

[Lee03] John M. Lee: Introduction to smooth manifolds, volume 218 of Graduate Texts in Mathematics. Springer-Verlag, New York, 2003.

[MS99] Dusa McDuff, Dietmar Salamon: Introduction to Symplectic Topology. Oxford: Oxford University Press, 1999.

[PS68] Harry Pollard and Donald G. Saari: Singularities of the $n$-body problem. I. Arch. Rational Mech. Anal., 30:263-269, 1968.

[Saa71] Donald G. Saari: Improbability of collisions in Newtonian gravitational systems. Trans. Amer. Math. Soc., 162:267-271; erratum, ibid. 168 (1972), 521, 1971.

[Saa73] Donald G. Saari: Improbability of collisions in Newtonian gravitational systems. II. Trans. Amer. Math. Soc., 181:351-368, 1973.

[Su] John M. Sullivan: Curves of finite total curvature. Discrete Differential Geometry 38,137-161, 2008 